OPEN ACCESS

Edited by:

Chaozheng Li,

Sun Yat-Sen University, China

Reviewed by:

WeiWei Li,

East China Normal University, China

Jing Xing,

Ocean University of China, China

*Correspondence:

Fangyi Chen

chenfangyi@xmu.edu.cn

Specialty section:

This article was submitted to Comparative Immunology, a section of the journal

Frontiers in Immunology

Received: 31 January 2021 Accepted: 16 March 2021 Published: 09 April 2021

Citation:

Qiu W, Chen F, Chen R, Li S, Zhu X, Xiong $M$ and Wang $K$-J (2021) A New

C-Type Lectin Homolog SpCTL6

Exerting Immunoprotective Effect and

Regulatory Role in Mud Crab

Scylla paramamosain.

Front. Immunol. 12:661823.

doi: 10.3389/fimmu.2021.661823

\section{A New C-Type Lectin Homolog SpCTL6 Exerting Immunoprotective Effect and Regulatory Role in Mud Crab Scylla paramamosain}

\author{
Wanlei Qiu ${ }^{1}$, Fangyi Chen ${ }^{1,2,3 *}$, Roushi Chen ${ }^{1}$, Shuang Li $^{1}$, Xuewu Zhu ${ }^{1}$, Ming Xiong ${ }^{1,2,3}$ \\ and Ke-Jian Wang ${ }^{1,2,3}$
}

1 State Key Laboratory of Marine Environmental Science, College of Ocean \& Earth Sciences, Xiamen University, Xiamen, China, ${ }^{2}$ State-Province Joint Engineering Laboratory of Marine Bioproducts and Technology, College of Ocean \& Earth Sciences, Xiamen University, Xiamen, China, ${ }^{3}$ Fujian Innovation Research Institute for Marine Biological Antimicrobial Peptide Industrial Technology, College of Ocean \& Earth Sciences, Xiamen University, Xiamen, China

C-type lectin (CTL), a well-known immune-related molecule, has received more and more attention due to its diverse functions, especially its important role in development and host defense of vertebrate and invertebrate. Since the research on crab CTLs is still lack, we screened a new CTL homolog, named SpCTL6 from mud crab Scylla paramamosain. The full-length cDNA sequence of SpCTL6 was 738 bp with a 486 bp of ORF, and the deduced amino acids were 161 aa. SpCTL6 was predicted to have a 17 aa signal peptide and its mature peptide was 144 aa (MW $16.7 \mathrm{kDa}$ ) with pl value of 5.22. It had typical CTL structural characteristics, such as a single C-type lectin-like domain, 4 conserved cysteines, similar tertiary structure to that of vertebrate CTLs and a mutated $\mathrm{Ca}^{2+}$ binding motif Gln-Pro-Thr (QPT), clustering into the same branch as the crustacean CTLs. SpCTL6 was highly expressed in the entire zoeal larval stages and widely distributed in adult crab tissues with the highest transcription level in testis. During the molting process of juvenile crabs, the expression level of SpCTL6 was remarkably increased after molting. SpCTL6 could be significantly upregulated in two larval stages (Z1 and megalopa) and adult crab testis under immune challenges. Recombinant SpCTL6 (rSpCTL6) was successfully obtained from eukaryotic expression system. rSpCTL6 exhibited binding activity with PAMPS (LPS, lipoteichoic acid, peptidoglycan, and glucan) and had a broad spectrum bacterial agglutination activity in a $\mathrm{Ca}^{2+}$-dependent manner. In addition, rSpCTL6 could enhance the encapsulation activity of hemocytes and has no cytotoxic effect on hemocytes. Although rSpCTL6 had no bactericidal activity on Vibrio alginolyticus, rSpCTL6 treatment could significantly reduce the bacterial endotoxin level in vitro and greatly improved the survival of $S$. paramamosain under $V$. alginolyticus infection in vivo. The immunoprotective effect of rSpCTL6 might be due to the regulatory role of rSpCTL6 in immune-related genes and immunological parameters. Our study 
provides new information for understanding the immune defense of mud crabs and would facilitate the development of effective strategies for mud crab aquaculture disease control.

Keywords: C-type lectin, SpCTL6, development, immunoprotective effect, regulatory role

\section{INTRODUCTION}

Mud crabs, Scylla paramamosain, due to their deliciousness, rich nutrition and high commodity value, have become an important global economic fishery species, which are mainly distributed in the Western Indo-Pacific (1). The capture fishery of mud crabs has been unable to meet the market demand, and the mud crab aquaculture industry has emerged and developed rapidly $(2,3)$. In 2018, the aquaculture production of mud crabs in China (157,712 tons) far exceeded that of the captured ones $(79,444$ tons), making it the most productive crab among marine aquaculture crabs in China (4). Although the total production of mud crabs has increased significantly in recent years, the artificial breeding technology of mud crab seedlings is still one of the main scientific and technological problems that have not been solved yet. High mortality occurs frequently during the developmental stage, especially the metamorphosis stage of the larvae [the survival rate was only about $10 \%$ from hatching to first-stage crabs (5)], the molting stage of juvenile crabs, and the reproductive molting process, which is closely related to the vulnerability of $S$. paramamosain to pathogens in these processes (6-8). In the past few decades, the research on mud crab immune system has made considerable progress, and many efforts have been made to encourage the development of mud crab aquaculture, such as the use of probiotics, immune-stimulants, and natural products, as reviewed in-depth previously (6). However, these strategies are limited, and we are still far away from understanding the molecular mechanisms of mud crab immunity.

C-type lectin (CTL), a well-known immune-related molecule, has received more and more attention due to its diverse functions, especially its important role in development and host defense of vertebrate and invertebrate $(9,10)$. Having at least one CTL-like domain (CTLD) is an indispensable feature of the CTLs superfamily (11). Most reported CTLs showed $\mathrm{Ca}^{2+}$ dependent carbohydrate binding activity and were considered as pattern recognition receptors (PRRs), with a few exceptions (9). Since the first CTL was discovered in bovine in 1906, more than a thousand CTL members have been identified in Metazoa, with dozens or even hundreds of CTL homologous genes in their genomes $(10,12)$. In vertebrate, these highly diverse CTLs have been demonstrated to play an important role in the regulation of innate and adaptive immunity, through non-self-recognizing or mediating intercellular communication and interaction, and could act as effector molecules exhibiting directly antibacterial activity $(9,13,14)$. And CTLs with single CTLD in invertebrate are also well-studied, with insects and crustaceans as representatives (10). Similarly, the immune function of CTLs in invertebrates is evolutionarily conserved. They participate in both cellular and humoral immunity of invertebrates, such as promoting hemocyte phagocytosis, mediating hemocyte encapsulation, nodule formation, activating the phenol oxidase (PO) system and ultimately leading to melanization, direct bactericidal activity, regulating immune signal transduction pathways and the expression of immune effectors etc., as summarized in detail previously $(10,15,16)$.

Currently, several crustacean genomes have been sequenced and assembled, such as the Pacific white shrimp Litopenaeus vannamei (17), the marbled crayfish Procambarus virginalis (18), the Chinese mitten crab Eriocheir sinensis (19), and the swimming crab Portunus trituberculatus (20), which would greatly facilitate the research on crustacean CTLs. So far, more than 90 crustacean CTLs have been reported. Among them, significant progress has been made in the study of shrimp CTLs, whose immune-related functions, molecular mechanisms involved in host-virus (WSSV) interactions, and the expression regulatory mechanism have been thoroughly discussed, providing a valuable reference for the research on other crustacean CTLs $(15,16)$. In crabs, numerous studies in CTLs have also been conducted, such as the E. sinensis CTLs (21-26), and the $P$. trituberculatus CTLs (27-30).

For the mud crab $S$. paramamosain, a total of six CTLs (SpCTLs) have been reported, including SpLec1 and SpLec2 (31), Sp-lectin3 and Sp-lectin4 (32), SpCTL-B (33), SpCTL5 (34). Analysis of SpCTLs coding sequences showed that they all contained only one CTLD, and four of them had signal peptides (SpLec1, SpLec2, Sp-lectin3 and Sp-lectin4), which is common in most crustacean CTLs. Conserved carbohydrate recognition motifs have also been found in SpCTLs, such as QPD (Gln-Pro-Asp) in Sp-lectin4 (32), EPD (Glu-Pro-Asp) in SpCTL-B (33). All SpCTLs were predominantly distributed in the hepatopancreas of adult mud crabs, which is an important immune organ. Among them, SpLec1 and SpLec2, Splectin3 and Sp-lectin4 were also distributed in the embryo and larval stage of crabs, indicating that they might play an important role in the development of crabs. Immune stimulations [such as Vibrio parahaemolyticus, Vibrio alginolyticus, LPS, Poly (I:C)] could significantly induce the expression of SpCTLs. Recombinant SpCTL protein expression was only performed in SpCTL-B (rSpCTL-B) and SpCTL5 (rSpCTL5) using prokaryotic expression system. In vitro functional studies showed that both rSpCTL-B and rSp-CTL5 showed bacterial agglutination activity in a $\mathrm{Ca}^{2+}$-dependent manner, while only rSpCTL-B exhibited potent antibacterial activity. When the expression of SpCTL-B was inhibited by RNAi, the bacterial load in hemocytes increased significantly, and several immune-related genes [including SpSTAT and five antimicrobial peptides (AMPs)] were downregulated, indicating its role in the immune response and regulation (33). Although some progress has been made in $S$. paramamosain CTLs, the functions and molecular mechanisms 
of SpCTLs are still largely unknown. Therefore, more SpCTLs are expected to be screened and more in-depth studies are needed.

In this study, a new CTL homolog, named SpCTL6 was identified from mud crab S. paramamosain. The full-length cDNA sequence was obtained. The expression profiles of SpCTL6 gene was analyzed by absolute quantitative PCR (qPCR) and relative qPCR. The recombinant protein (rSpCTL6) was expressed in Pichia pastoris eukaryotic expression system and purified by affinity chromatography. The binding, hemagglutination, agglutination, and encapsulation activity analysis of rSpCTL6 were performed in vitro. In addition, the cytotoxicity and the endotoxin level of $V$. alginolyticus after rSpCTL6 treatment were determined. The immunoprotective effect and the regulatory role of rSpCTL6 in S. paramamosain challenged with $V$. alginolyticus were evaluated through analyzing the survival rate of crabs, the bacterial clearance ability in gills and hepatopancreas, the expression of immune-related genes and the enzymatic activity of immunological parameters including phenol oxidase (PO), lysozyme (LZM), peroxidase (POD), superoxide dismutase (SOD), alkaline phosphatase (AKP), and acid phosphatase (ACP). This study aims to enrich the knowledge of crab CTLs through in-depth study of its function, immune protective effect and related mechanism, providing important information for understanding the immune defense of mud crabs and facilitating the development of effective strategies for mud crab aquaculture disease control.

\section{MATERIALS AND METHODS}

\section{Animals, Sample Collection and Immune Challenge}

Adult male and female mud crabs (S. paramamosain) weighing about $300 \pm 30 \mathrm{~g}$ were purchased from a crab farm in Zhangzhou City, Fujian Province, China. The hemocytes were prepared as previously described (35). Multiple tissues of crabs $(n=5)$ were sampled (including gills, hepatopancreas, midgut, eyestalks, subcuticular epidermis, heart, muscle, stomach, thoracic ganglion, female crab gonadal tissues (ovaries, spermatheca, and reproductive duct) and male crab gonadal tissues (testis, anterior vas deferens, seminal vesicle, posterior vas deferens, ejaculation ducts, posterior ejaculation ducts) and ready for total RNA extraction. The various stages of crab development, including embryonic stages (Em1-Em5), zoeal larval stages (Z1-Z5), megalopa stage, three juvenile stages (JU1, JU2, and JU3), the molting process of juvenile crabs including premolt and postmolt samples (JU1 developed to JU2, JU2 developed to JU3) $(n=5)$ were collected from a crab breeding farm in Beihai City, Guangxi Province, China. The different larval stages have their unique features, which can be easily identified as described in detail previously $(36,37)$.

Two larval stages (Z1 larvae and megalopa) were selected for immune challenge experiments. Thousands of Z1 larvae or megalopa were randomly divided into 6 petri dishes $(1.5 \mathrm{~L})$, and $700 \mathrm{~mL}$ of sterilized sea water was added. The experiment included three different groups: the control group (sea water), the bacterial infection group $\left(5 \times 10^{6}\right.$ colony-forming units (CFU)/ $\mathrm{mL} V$. alginolyticus), and the LPS stimulation group $[200 \mathrm{ng} / \mathrm{mL}$ LPS (Sigma, USA)]. The larvae were sampled at $3 \mathrm{~h}, 6 \mathrm{~h}, 9 \mathrm{~h}, 12 \mathrm{~h}$, and $24 \mathrm{~h}$ after challenge. Five biological parallel samples $(\mathrm{n}=5)$ were set up at each time point (each parallel sample contained dozens of larvae). They were immediately put into liquid nitrogen and then stored at $-80^{\circ} \mathrm{C}$ for later use.

The purchased adult male crabs $(300 \pm 30 \mathrm{~g})$ were acclimated at $23 \pm 2{ }^{\circ} \mathrm{C}$ for several days before the challenge experiment. Ninety crabs were randomly divided into three groups (30 crabs for each group), including the control group, the $V$. alginolyticus infection group $\left(3 \times 10^{6} \mathrm{CFU} / \mathrm{crab}\right)$, and the LPS stimulation group $(0.5 \mathrm{mg} / \mathrm{kg} \cdot \mathrm{crab})$. For the control group, $100 \mu \mathrm{L}$ of crab saline (4.96 mM NaCl, $9.52 \mathrm{mM} \mathrm{KCl}, 0.8 \mathrm{mM} \mathrm{MgSO}_{4}, 16.2 \mathrm{mM}$ $\mathrm{CaCl}_{2}, 0.84 \mathrm{mM} \mathrm{MgCl}_{2}, 5.95 \mathrm{mM} \mathrm{NaHCO}, 20 \mathrm{mM}$ HEPES, pH 7.4) was injected. Testis $(n=5)$ were sampled in each group at 3,6 , $12,24,48$ and $72 \mathrm{~h}$ post-injection (hpi) and then stored in $-80^{\circ} \mathrm{C}$ for later use.

Total RNA of the collected samples were extracted using Trizol reagent (Thermo Fisher Scientific, USA) according to the manufacturer's instructions. RNA quality was evaluated by NanoDrop 2000 spectrophotometer (Thermo Fisher Scientific, USA), and PrimeScript ${ }^{\mathrm{TM}}$ RT reagent kit with gDNA eraser (TaKaRa, Japan) was used for cDNA synthesis.

\section{Cloning of the Full-Length cDNA Sequence of SpCTL6}

The cDNA of adult male crab testis was synthesized as described above. The $5^{\prime}$ and $3^{\prime}$ RACE cDNA were prepared using SMARTer ${ }^{\circledR}$ RACE 5'/3' Kit (Clontech, USA). The coding sequence (CDS) of SpCTL6 was obtained from the transcriptome database established by our laboratory. A pair of primers (SpCTL6-CDS-F and SpCTL6-CDS-R, as shown in Table 1) were designed to amplify SpCTL6 CDS and testis cDNA was used as a template. The PCR was carried out as follows: $95^{\circ} \mathrm{C}, 5 \mathrm{~min} ; 30$ cycles of $95^{\circ} \mathrm{C}, 30 \mathrm{~s}, 62^{\circ} \mathrm{C}, 30 \mathrm{~s}, 72^{\circ} \mathrm{C} 1$ min; $72^{\circ} \mathrm{C}$ for 5 minute. And primers (SpCTL6-5-R1, SpCTL6-5R2, SpCTL6-3-F1, SpCTL6-3-F2, as shown in Table 1) were synthesized to amplify the $5^{\prime}$ and $3^{\prime}$ cDNA ends of SpCTL6 using nested PCR and touchdown PCR. The first round of PCR used pairs of primers (SpCTL6-5-R1/Long primer for 5' RACE PCR, SpCTL6-3-F1/Long primer for 3' RACE PCR) and the second round of PCR used SpCTL6-5-R2/Short primer for 5' RACE PCR, SpCTL6-3-F2/Short primer for 3' RACE PCR, respectively. The Long primer and Short primer were provided by SMARTer ${ }^{\circledR}$ RACE 5'/3' Kit. The touch down PCR procedure was performed as followed: $95^{\circ} \mathrm{C}, 5 \mathrm{~min} ; 30$ cycles of $95^{\circ} \mathrm{C}, 30 \mathrm{~s}, 68^{\circ} \mathrm{C}-0.5 /$ cycle, $30 \mathrm{~s}, 72^{\circ} \mathrm{C}, 2 \mathrm{~min} ; 72^{\circ} \mathrm{C}, 10 \mathrm{~min} ; 16^{\circ} \mathrm{C} 5 \mathrm{~min}$. The PCR products were then purified and sequenced by Sangon Biotech (Shanghai) Co., Ltd.

\section{Bioinformatics and Phylogenetic Analysis of SpCTL6}

The homology of the SpCTL6 gene sequence with other sequences was analyzed by the basic local alignment search 
TABLE 1 | Sequences of primers used in this study.

\begin{tabular}{|c|c|}
\hline Primer name & Primer sequences $\left(5^{\prime}-3^{\prime}\right)$ \\
\hline SpCTL6-CDS-F & ATGCTGCGCGTGTACTGCCTCCTCC \\
\hline SpCTL6-CDS-R & TCAGAAGGCGTGGACCTCGTTCTGA \\
\hline SpCTL6-5-R1 & AGCCACGTGGTTGTAATCGAA \\
\hline SpCTL6-5-R2 & AAGCACATGTAGTTGAGCACG \\
\hline SpCTL6-3-F1 & TTGACGACCGTGCCCTTAG \\
\hline SpCTL6-3-F2 & GCCCTTAGCCCCAACTCTATT \\
\hline Long primer & $\begin{array}{l}\text { CTAATACGACTCACTATAGGGCAAGCA } \\
\text { GTGGTATCAACGCAGAGT }\end{array}$ \\
\hline Short primer & CTAATACGACTCACTATAGGGC \\
\hline SpCTL6-qPCR-F & ACGACGCCTCCTGGTITGG \\
\hline SpCTL6-qPCR-R & GGCGTGGACCTCGTTCTGAC \\
\hline SpSOD-F & GGGGATGGGAAACAACTCTGGAT \\
\hline SpSOD-R & GGTGCCTTGGTTAAATACACGGTGC \\
\hline SpALF6-F & TCAAGGGAGACGTGTGGTGC \\
\hline SpALF6-R & TGGCGAAGTCTGCGATAGCC \\
\hline SpCrustin3-F & АCCTGCCTGGCCATTACGTG \\
\hline SpCrustin3-R & CCCACCACAGGGAGTGTTGC \\
\hline GADPH-qPCR-F & CTCCACTGGTGCCGCTAAGGCTGTA \\
\hline GADPH-qPCR-R & CAAGTCAGGTCAACCACGGACACAT \\
\hline rSpCTL6-EcoRI-F & CGGAATTCGCGTGCCCTGCCCCCTTTGT \\
\hline \multirow[t]{2}{*}{ rSpCTL6-Notl-R } & ATAAGAATGCGGCCGCTCAGTGGTGGTG \\
\hline & GTGGTGGTGGAAGGCGTGGACCTCGTTC \\
\hline
\end{tabular}

tool (BLAST) of NCBI (http://www.ncbi.nlm.nih.gov/blast). The signal peptide and conserved domain of SpCTL6 were predicted by SignalP 4.1 Server (http://www.cbs.dtu.dk/services/SignalP/) and SMART database (http://smart.embl-heidelberg.de/), respectively. Expasy (http://web.expasy.org/protparam) was used to calculate the theoretical molecular weight (MW) and isoelectric point (pI) of SpCTL6. The secondary and tertiary structures of SpCTL6 were predicted by UCL (http://bioinf.cs. ucl.ac.uk/psipred/) and SWISS-MODEL (https://swissmodel. expasy.org/), respectively. Multiple sequence alignment between SpCTL6 and other CTLs was performed using Clustal $\mathrm{X} 2.1$ software. The phylogenetic tree was constructed using the neighbor joining method of MEGA 6.0, and the reliability was evaluated by 1000 bootstraps.

\section{Quantitative Real-Time PCR Analysis of the Expression Profiles of SpCTL6}

The expression profiles of SpCTL6 gene in various adult crab tissues and different developmental stages were determined by absolute quantitative real-time PCR (qPCR) and the expression changes of SpCTL6 during the molting stages of juvenile crabs and the response patterns of SpCTL6 gene to LPS and V. alginolyticus challenge were analyzed by relative qPCR. GAPDH gene of $S$. paramamosain (GenBank accession number: JX268543.1) was employed as the internal reference gene in relative qPCR assay. Gene-specific primers (SpCTL6-qPCR-F/SpCTL6-qPCR-R, GADPH-qPCR-F/GADPH-qPCR-R, listed in Table 1) were designed. The SpCTL6 CDS plasmid was used to generate its standard curve. qPCR was performed on Qtower 2.2 (Analytik Jena, Germany) as followed: $50^{\circ} \mathrm{C}$ for $2 \mathrm{~min}, 95^{\circ} \mathrm{C}$ for $10 \mathrm{~min}, 40$ cycles of $95{ }^{\circ} \mathrm{C}$ for $15 \mathrm{~s}, 60^{\circ} \mathrm{C}$ for $1 \mathrm{~min}$. The absolute copy numbers of SpCTL6 gene were calculated according to the linear regression of the standard curve. The data for relative qPCR was analyzed using the algorithm of the $2^{-\Delta \Delta \mathrm{Ct}}$ method (38).

\section{Expression and Purification of Recombinant SpCTL6 (rSpCTL6) in Pichia Pastoris Eukaryotic Expression System}

The mature peptide sequence of SpCTL6 was constructed into the pPIC9K vector using primers rSpCTL6-EcoRI-F/rSpCTL6NotI-R (as shown in Table 1). The recombinant plasmid was linearized by restriction enzyme $S a c \mathrm{I}$, and then transformed into competent $P$. pastoris cells by electroporation. A positive clone was picked and cultured to logarithmic growth phase at $28{ }^{\circ} \mathrm{C}$ in YPD medium ( $2 \%$ tryptone, $1 \%$ yeast extract, $2 \% \mathrm{D}$-glucose). Then the medium was replaced with BMGY medium (10 g yeast extract, $20 \mathrm{~g}$ tryptone were dissolved in $700 \mathrm{~mL}$ water, autoclaved for $20 \mathrm{~min}$, cooled to room temperature, and the following mixture were added: $100 \mathrm{~mL} 1 \mathrm{M}$ potassium phosphate buffer ( $\mathrm{pH}$ 6.0), $100 \mathrm{~mL} 10 \mathrm{YNB}, 100 \mathrm{~mL} 10 \%$ glycerine, $2 \mathrm{~mL}$ $500 \times$ biotin) until it cultured to logarithmic then $P$. pastoris cells were induced by $0.5 \%$ methanol in BMMY (dissolve $10 \mathrm{~g}$ yeast extract, $20 \mathrm{~g}$ tryptone in $700 \mathrm{~mL}$ water, autoclaviate for 20 min, cool to room temperature, add the following mixture: 100 $\mathrm{mL} 1 \mathrm{M}$ potassium phosphate buffer ( $\mathrm{pH} 6.0$ ), $100 \mathrm{~mL} 10 \mathrm{YNB}$, $100 \mathrm{~mL} 10 \%$ methanol, $2 \mathrm{~mL} 500 \times$ biotin). After 24 hours of induction, the supernatant was collected and dialyzed against buffer ( $50 \mathrm{mM}$ phosphate buffer, $50 \mathrm{mM} \mathrm{NaCl}, \mathrm{pH}$ 9.0). The target protein was purified by ÄKTA Pure system (GE Healthcare Life Sciences, USA) using a HisTrap ${ }^{\text {TM }}$ FF Crude column (GE Healthcare Life Sciences, USA). The purity of rSpCTL6 was analyzed by SDS-PAGE, and the sequence was confirmed by the Mass Spectrometry Center of the School of Life Sciences, Xiamen University. The protein concentration was determined by Bradford assay kit (Beyotime Institute of Biotechnology, China). All recombinant protein rSpCTL6 were stored at $-80^{\circ} \mathrm{C}$ for later use.

\section{Hemagglutination, Bacterial Agglutination, Binding, and Encapsulation Assay Hemagglutination Assay}

The blood of mouse was collected in a centrifuge tube containing sodium citrate. After washing 3 times with TBS buffer, the blood was re-suspended in TBS buffer at a concentration of $2 \%(\mathrm{v} / \mathrm{v})$, and then transferred to a 96-well type $\mathrm{V}$ hemagglutination plate. Four groups were set up (rSpCTL6 with or without $10 \mathrm{mM} \mathrm{CaCl}_{2}$ groups, TBS with $10 \mathrm{mM} \mathrm{CaCl} 2$ group, and BSA with10 mM $\mathrm{CaCl}_{2}$ group). rSpCTL6 was added at a series of dilution concentrations (from $0.75 \mu \mathrm{g} / \mathrm{mL}$ to $100 \mu \mathrm{g} / \mathrm{mL}$ ). An equal volume of BSA $(100 \mu \mathrm{g} / \mathrm{mL})$ and TBS with $10 \mathrm{mM} \mathrm{CaCl}_{2}$ were added as control groups. Hemagglutination was observed after incubation at $4^{\circ} \mathrm{C}$ for $1 \mathrm{~h}$. Each group had three biological parallels. The independent experiment was repeated at least three times.

\section{Bacterial Agglutination Assay}

Gram-positive bacteria (Staphylococcus aureus), and Gramnegative bacteria (Pseudomonas aeruginosa, Pseudomonas fluorescens, Aeromonas hydrophila, Vibrio harveyi, Vibrio fluvialis, $V$. parahaemolyticus, and $V$. alginolyticus) were selected to investigate the agglutination activity of rSpCTL6. All the bacteria were cultured to the logarithmic growth phase, 
washed 3 times with TBS, and re-suspended in $0.1 \mathrm{M} \mathrm{NaHCO}_{3}$ ( $\mathrm{pH}$ 9.0). They were then incubated with a final concentration of $0.1 \mathrm{mg} / \mathrm{mL}$ FITC (Sigma, USA) for $30 \mathrm{~min}$ at room temperature, and washed 3 times with TBS to remove unlabeled FITC. The concentration of the bacteria was adjusted to $10^{8} \mathrm{CFU} / \mathrm{mL}$. Four groups were set up (rSpCTL6 with or without $10 \mathrm{mM} \mathrm{CaCl}$ groups, TBS with $10 \mathrm{mM} \mathrm{CaCl}_{2}$ group, and BSA with $10 \mathrm{mM}$ $\mathrm{CaCl}_{2}$ group). The FITC-labeled bacteria were incubated with 10 $\mu \mathrm{L}$ of $\mathrm{rSpCTL} 6(40 \mu \mathrm{g} / \mathrm{mL})$ at room temperature in the dark for 1 h. An equal volume of BSA $(40 \mu \mathrm{g} / \mathrm{mL})$ and TBS with $10 \mathrm{mM}$ $\mathrm{CaCl}_{2}$ were added as control groups. The agglutination results were observed with an inverted fluorescence microscope (Zeiss, Germany). Each group had three biological parallels. The independent experiment was repeated at least three times.

\section{Binding Assay}

A modified enzyme-linked immune sorbent assay (ELISA) was carried out as previously described (39). Briefly, LPS, peptidoglycan (PGN), lipoteichoic acid (LTA) and glucan (GLU) were diluted with an ELISA coating solution to a working concentration of $20 \mu \mathrm{g} / \mathrm{mL}$, and then added to a 96well ELISA plate. The plate was coated overnight at $4^{\circ} \mathrm{C}$, blocked with $5 \%$ skim milk at $37^{\circ} \mathrm{C}$ for $2 \mathrm{~h}$, and then added with a series dilution of rSpCTL6 $(0 \sim 100 \mu \mathrm{g} / \mathrm{mL})$ at $37^{\circ} \mathrm{C}$ for $2 \mathrm{~h}$. There were 3 parallels for each concentration of protein. Mouse anti-His antibody (1:5000, prepared in $1 \%$ skim milk) was added and incubated at $37^{\circ} \mathrm{C}$ for $2 \mathrm{~h}$ followed by incubation with goat antimouse HRP antibody (1:5000). TMB solution was added and incubated at $37^{\circ} \mathrm{C}$ for $10-30 \mathrm{~min}$. The reaction was stopped with $2 \mathrm{M} \mathrm{H}_{2} \mathrm{SO}_{4}$. The absorbance at $450 \mathrm{~nm}$ was measured using a microplate reader (TECAN GENios, GMI, USA). Each group had three biological parallels. The independent experiment was repeated at least three times.

\section{Encapsulation Assay}

In order to evaluate the encapsulation activity of rSpCTL6, the NiNTA agarose beads [Senhui Microsphere Technology (Suzhou) Co., Ltd, China] was used. Four groups were also set up (rSpCTL6 with or without $10 \mathrm{mM} \mathrm{CaCl}$ groups, TBS with $10 \mathrm{mM} \mathrm{CaCl}_{2}$ group, and BSA with10 $\mathrm{mM} \mathrm{CaCl} 2$ group). The beads were first equilibrated in TBS, then incubated with different amounts of rSpCTL6 $(25 \mu \mathrm{g}-200 \mu \mathrm{g})$ at $4{ }^{\circ} \mathrm{C}$ overnight, and washed with TBS 3 times. A 24 -well culture plate was pre-coated with $1 \%$ agarose. The hemocytes were isolated from $S$. paramamosain as previously described (35), and added to the plate for at least $10 \mathrm{~min}$ to allow them settle down. The beads containing proteins were then added and incubated at $26^{\circ} \mathrm{C}$. Encapsulation was detected after 6 and $24 \mathrm{~h}$ under a light microscope (LEICA DMi1, Germany). Each group had three biological parallels. The independent experiment was repeated at least three times.

\section{Alginolyticus Endotoxin Assay}

The endotoxin level of $V$. alginolyticus was detected by the Toxin Sensor $^{\mathrm{TM}}$ Chromogenic LAL Endotoxin Assay Kit (GenScript, USA) following the manufacturer's instructions. V. alginolyticus was collected when reaching to the logarithmic growth phase and adjusted to a concentration of $10^{7} \mathrm{CFU} / \mathrm{mL}$. They were then incubated with different concentration of rSpCTL6 (10.5, 21, 42, $84 \mu \mathrm{M}$ ) at room temperature for $1 \mathrm{~h}$ and analyzed by a spectrophotometer at an absorbance of $545 \mathrm{~nm}$ (Agilent Technologies. Malaysia). Each concentration of rSpCTL6 treatment had three biological parallels. The independent experiment was repeated at least three times.

\section{Cytotoxicity Assay}

The hemocytes of adult crabs were primarily cultured as previously described $(40,41)$. About $1 \times 10^{5}$ cells/well were inoculated on a 96-well cell culture plate at $26^{\circ} \mathrm{C}$ and cultured overnight. Different concentration of rSpCTL6 $(3 \mu \mathrm{M}, 6 \mu \mathrm{M}, 12$ $\mu \mathrm{M}, 24 \mu \mathrm{M}, 48 \mu \mathrm{M})$ were added. After $24 \mathrm{~h}$ of incubation, the CellTiter $96^{\circledR}$ AQueous Kit (Promega) was used to evaluate the viability of hemocytes. Each concentration of rSpCTL6 treatment had three biological parallels. The independent experiment was repeated at least three times.

\section{Effect of rSpCTL6 on Mud Crab S. Paramamosain Infected by V. Alginolyticus Mortality Test}

To investigate whether rSpCTL6 has an immunoprotective effect on S. paramamosain, a bacterial challenge experiments was performed in male mud crabs $(100 \pm 10 \mathrm{~g})$. V. alginolyticus $\left(2 \times 10^{7} \mathrm{CFU} \mathrm{crab}^{-1}\right)$ were first injected into the base of the right fourth leg of the crabs $(n=105)$. After bacterial injection, 105 crabs were randomly divided into three groups $(n=35)$ (control group, $10 \mu \mathrm{g} \mathrm{crab}^{-1} \mathrm{rSpCTL6}$ treatment group and $20 \mu \mathrm{g} \mathrm{crab}^{-1}$ rSpCTL6 treatment group). Two hours later, rSpCTL6 (10 $\mu \mathrm{g}$ $\mathrm{crab}^{-1}$ or $20 \mu \mathrm{g} \mathrm{crab}^{-1}$ ) was injected, and an equal volume of the crab saline was injected as the control group. Survival crabs were recorded at different time point $(3 \mathrm{~h}, 6 \mathrm{~h}, 9 \mathrm{~h}, 24 \mathrm{~h}, 36 \mathrm{~h}, 48 \mathrm{~h}, 60$ h, $72 \mathrm{~h}, 96 \mathrm{~h}, 120 \mathrm{~h}$ ), and a mortality curve was drawn by GraphPad Prism 8.3.0 version. The independent experiment was repeated twice.

\section{Bacterial Load Assay}

The bacterial load in two crab tissues (gills and hepatopancreas) of different groups (control group and $20 \mu \mathrm{g} \mathrm{crab}^{-1}$ rSpCTL6 treatment group) was determined at selected time points after treatment ( $3 \mathrm{~h}, 6 \mathrm{~h}$, and $24 \mathrm{~h}$ ). Briefly, gills and hepatopancreas $(n=3)$ were sampled from experimental crabs (treated with crab saline or $20 \mu \mathrm{g} \mathrm{crab}^{-1}$ rSpCTL6 after $2 \mathrm{~h}$ of bacterial injection), and approximately $100 \mathrm{mg}$ of each tissue was homogenized in $1 \mathrm{~mL}$ of PBS solution, then diluted to an appropriate concentration, and spread on an agar plate [containing $2216 \mathrm{E}$ cultured medium (Qingdao Hope Bio Tech., China)], and cultured at $28{ }^{\circ} \mathrm{C}$ overnight. The bacterial $\mathrm{CFU}$ of all plates were counted and recorded. Each sample had three biological parallels and three different dilutions.

\section{Enzymatic Activity Assay}

The gills and hepatopancreas were sampled $(n=3)$ and homogenized as described above and various enzymatic activity assays were performed according to the manufacturer's instructions of the corresponding protease detection kits 
[including PO, lysozyme (LZM), peroxidase (POD), superoxide dismutase (SOD), alkaline phosphatase (AKP), acid phosphatase (ACP) assays (Nanjing Jiancheng, China)].

\section{Immune-Related Genes Expression Analysis}

Several immune-related genes were selected to study their expression pattern after treatment (including signaling pathway genes ( $S p$ Toll2, Relish, SpDorsal, STAT), AMPs [SpCrustin3, SpCrustin5, SpALF2, SpALF6), cytokine gene (LITAF), antioxidant gene (SpSOD)]. The specific primers and genbank accession numbers for these genes were summarized in Tables $\mathbf{1}$ and $\mathbf{S 1}$. Relative qPCR was performed as described above.

\section{Statistical Analysis}

GraphPad Prism 8.0 Software was applied for analyzing relative qPCR assay and enzymatic activity assay using multiple t test one per row. The mortality curve was drawn by GraphPad Prism 8.0 Software and analyzed using the Kaplan-Meier Log rank test. Difference was considered as significant at $p<0.05$. All data were displayed as mean \pm standard deviation (SD).

\section{RESULTS}

\section{Sequence and Structure Analysis of SpCTL6}

The full-length cDNA sequence of SpCTL6 was 738 bp with a $486 \mathrm{bp}$ of ORF, and the deduced amino acids was 161 aa (Figure 1A). SpCTL6 was predicted to have a 17 aa signal peptide and its mature peptide was 144 aa (MW $16.7 \mathrm{kDa}$ ) with pI value of 5.22 , which contained a conserved CTLD (Figures 1A, B). Its tertiary structures were predicted, which has a double-loop structure, 2 $\alpha$-helices and $5 \beta$-sheets (Figure 1C). The phylogenetic analysis showed that SpCTL6 was clustered into the same branch as the crustacean CTLs (Figure 1D). Multiple sequences alignment analysis showed that it has 4 conserved cysteines and a GlnPro-Thr (QPT) motif (Figure 1E).

\section{The Expression Profiles of SpCTL6}

Based on qPCR analysis, SpCTL6 mRNA was at a relatively low level in the embryonic stage (Em1-Em4) of S. paramamosain. Its copy numbers gradually increased from the Em5 stage, reached the highest levels in the $\mathrm{Z} 1$ stage (about $1 \times 10^{6}$ copies $/ \mu \mathrm{L}$ ), maintained a high level (over $0.7 \times 10^{6}$ copies $/ \mu \mathrm{L}$ ) throughout the zoea larval stage, and then gradually reduced from the megalopa stage to juvenile crabs (Figure 2A). During the molting process of juvenile crabs, the expression of SpCTL6 was significantly upregulated after molting (JU1 developed to JU2, and JU2 developed to JU3) (Figure 2B). As reported, high mortality occurs frequently during the developmental stages, especially the metamorphosis stage of the larvae (5). Among them, $\mathrm{Z} 1$ is the larvae that has just hatched from embryo, and megalopa is the last larval stage undergoing metamorphosis from zoeal stage, which are two important metamorphosis stages. Therefore, we chose these two stages as representatives to investigate the immune response of SpCTL6 in the larval stages. After LPS stimulation, in the Z1 stage of $S$. paramamosain, SpCTL6 was significantly down-regulated at 3 $\mathrm{h}$ and up-regulated at $24 \mathrm{~h}$, while the expression of SpCTL6 did not show any change under the challenge of $V$. alginolyticus (Figures 2C, E). In the megalopa stage, the transcription level of SpCTL6 mRNA was remarkably increased at $12 \mathrm{~h}$ after LPS or bacterial challenge (Figures 2D, F).

The absolute qPCR results also showed that SpCTL6 was widely distributed in various tissues of male and female adult crabs, with the highest expression level in the testis of male adult crabs (Figures 3A, B). When adult male crabs were injected with LPS or $V$. alginolyticus, the expression of SpCTL6 was significantly down-regulated at $3 \mathrm{~h}$ and up-regulated at $72 \mathrm{~h}$ after LPS stimulation, and up-regulated at $3 \mathrm{~h}$ after bacterial challenge (Figures 3C, D).

\section{Recombinant SpCTL6 (rSpCTL6) Obtained From $P$. pastoris Eukaryotic Expression System}

We successfully obtained recombinant SpCTL6 (rSpCTL6) from the $P$. pastoris eukaryotic expression system. SDS-PAGE analysis showed that a specific positive band appeared at a position slightly larger than the $15 \mathrm{kDa}$ marker, corresponding to the predicted mature peptide size $(16.7 \mathrm{kDa})$, and the purity of rSpCTL6 was high as shown in Figure 4A. In addition, the results from the mass spectrometry also confirmed that the purified protein was the target protein rSpCTL6 (Figure S1).

\section{rSpCTL6 Showed Hemagglutination, Bacterial Agglutination, Binding, and Encapsulation Activity In Vitro}

For the type $\mathrm{V}$ hemagglutination plate used in hemagglutination assay, if the erythrocytes are observed to settle at the bottom of the plate, it indicates that the reaction is negative, that is, the erythrocytes have not agglutinated. The results showed that in the presence of $10 \mathrm{mM} \mathrm{Ca}^{2+}$, rSpCTL6 at a low concentration of $12.5 \mu \mathrm{g} / \mathrm{mL}$ could cause significant agglutination of mouse erythrocytes, not to mention higher concentrations $(25,50$, and $100 \mu \mathrm{g} / \mathrm{mL}$ ) (Figure 4B). In the $0 \mathrm{mM} \mathrm{Ca}^{2+}$ group and the control group, obvious sedimentation of erythrocytes was observed (Figure 4B). Therefore, the hemagglutination activity of rSpCTL6 was $\mathrm{Ca}^{2+}$ dependent. Similarly, rSpCTL6 could agglutinate all the selected bacteria (including several Vibrio species commonly found in aquaculture) in a $\mathrm{Ca}^{2+}$-dependent manner (Figure 4D). While the control groups (TBS and BSA) and rSpCTL6 without $\mathrm{CaCl}_{2}$ group could not induce agglutination of tested bacteria.

As shown in Figure 4C, rSpCTL6 had binding affinity to several tested microbial surface molecules (LPS, LTA, PGN, and $\mathrm{GLU})$. The calculated apparent dissociation constants $(\mathrm{Kd})$ were $1.876 \mu \mathrm{M}$ for LTA, $2.160 \mu \mathrm{M}$ for LPS, $2.804 \mu \mathrm{M}$ for PGN, 2.957 $\mu \mathrm{M}$ for GLU, respectively, which indicated that rSpCTL6 had the highest binding affinity for LTA.

rSpCTL6 pre-coated with Ni-NTA agarose beads was used to perform the encapsulation assay in vitro. The results showed that 


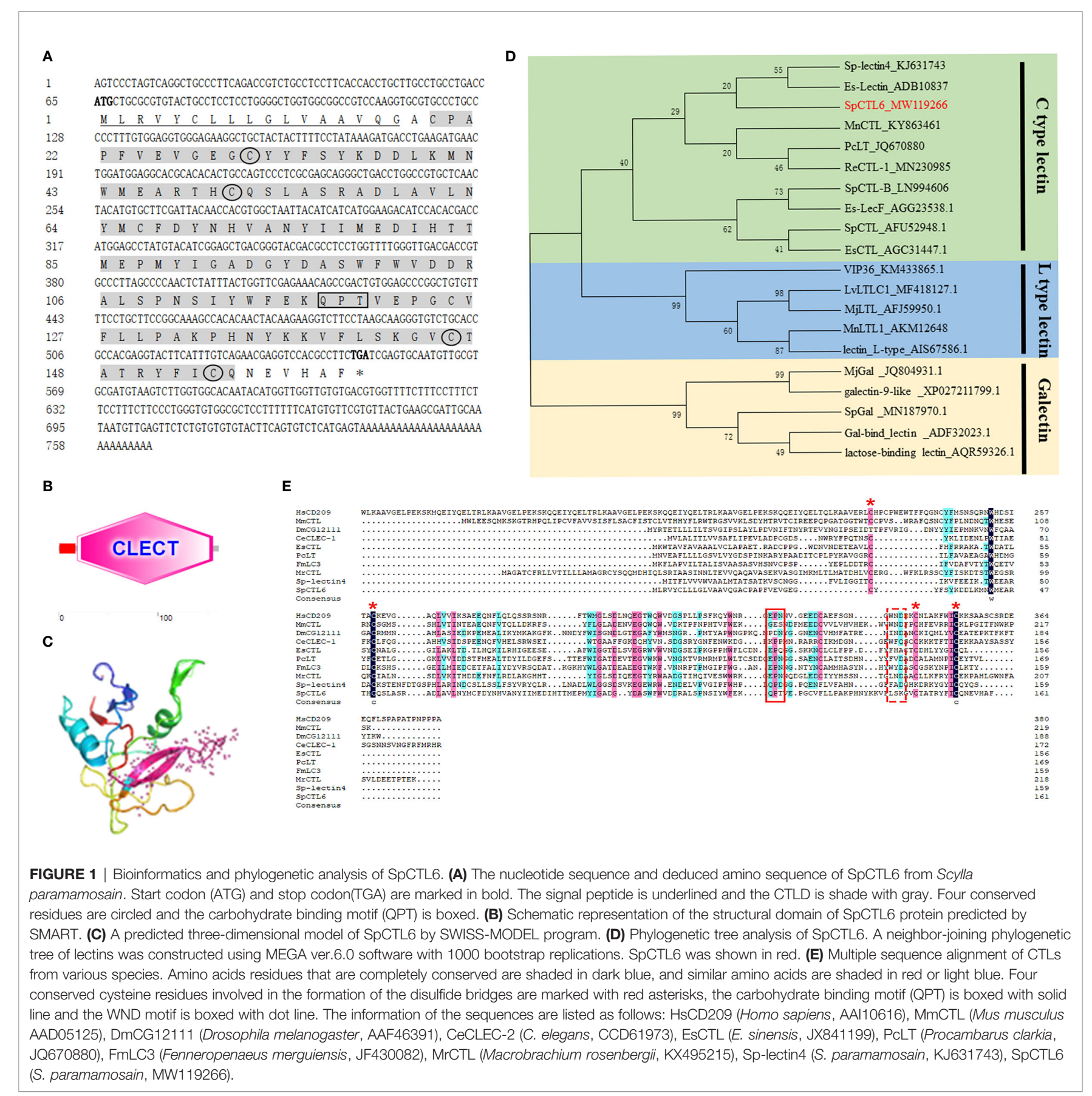

there was no obvious hemocytes encapsulating around the beads in the control groups and the encapsulation ratio was very low (TBS and BSA, with or without $\mathrm{Ca}^{2+}$ group) (Figures 5A, B). While in $200 \mu \mathrm{g}$ rSpCTL6 treatment group, after $6 \mathrm{~h}$ of incubation, dozens of hemocytes were recruited around the beads, the color of the beads became light dark, and the encapsulation ratio was about $7 \%$ (Figures 5A, B). Especially, in the presence of $10 \mathrm{mM} \mathrm{Ca}^{2+}$, $200 \mu \mathrm{g}$ rSpCTL6 treatment for $6 \mathrm{~h}$ resulted in an encapsulation ratio of about $98 \%$ and the beads color became black dark (Figures 5A, B). The encapsulation ratio of $24 \mathrm{~h}$ rSpCTL6 treatment without or with $\mathrm{Ca}^{2+}$ reached about $40 \%$ and $99.6 \%$, respectively (Figures 5A, B). And the encapsulation activity of rSpCTL6 exhibited in a dose-dependent manner (Figure S2).

\section{rSpCTL6 Had No Cytotoxicity on Hemocytes of S. Paramamosain and Could Reduce the V. Alginolyticus Endotoxin Level In Vitro}

The cytotoxicity of rSpCTL6 on hemocytes of S. paramamosain was evaluated. The results showed that different concentrations 


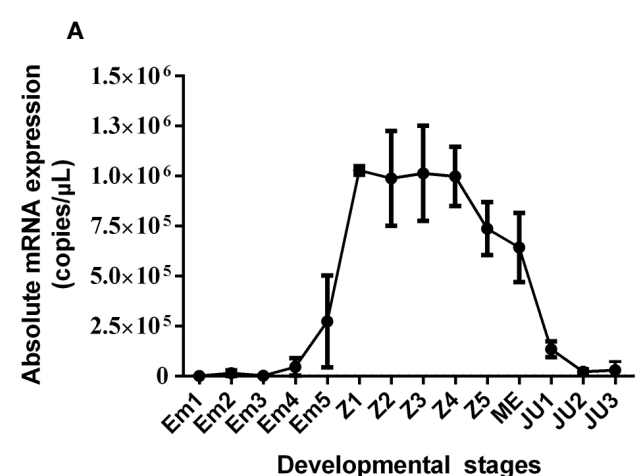

C

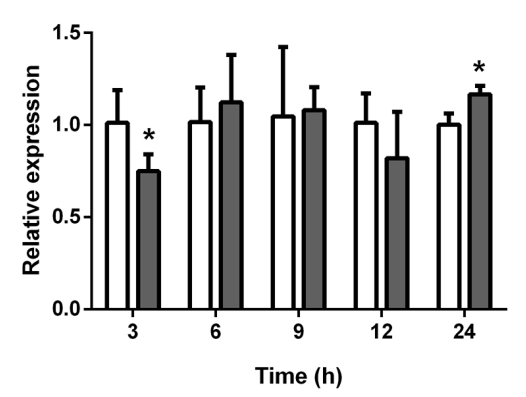

E

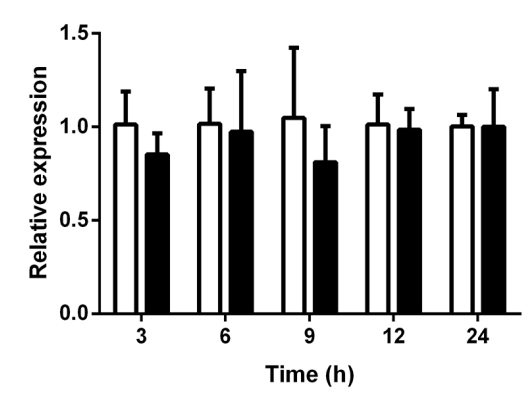

B

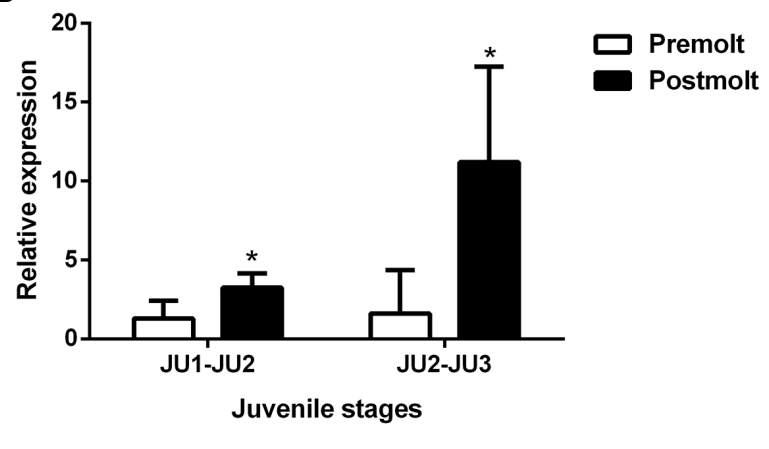

D Megalopa
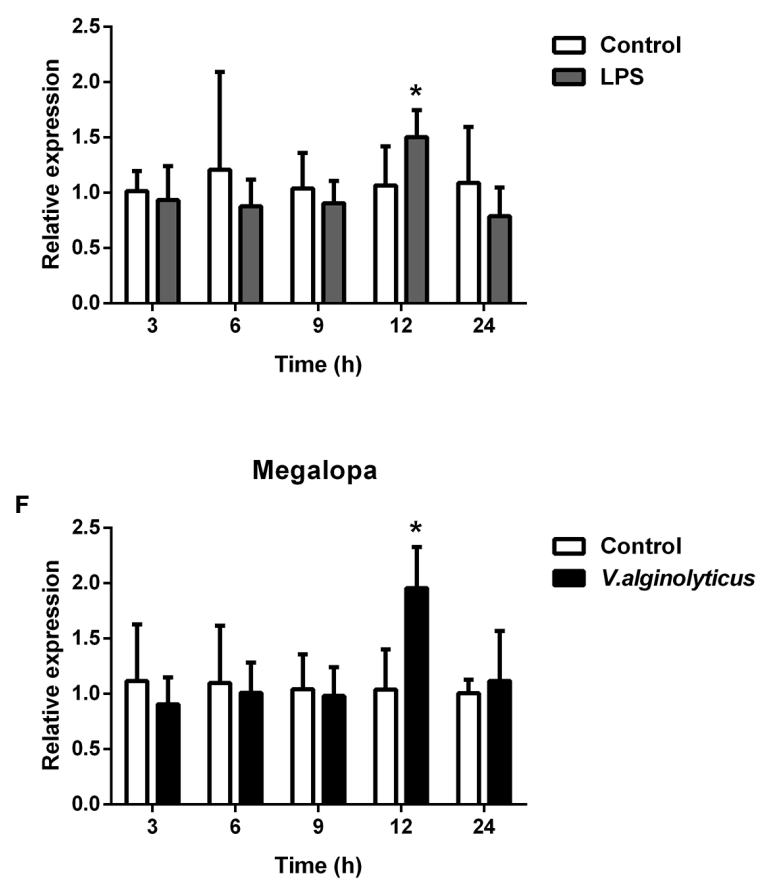

FIGURE 2 | Expression profiles of SpCTL6 gene in the developmental stages of S. paramamosain. (A) The expression of SpCTL6 in the different developmental stages of $S$. paramamosain was determined by absolute qPCR ( $n=5)$. Em1-Em5: embryonic stage 1-5; Z1-Z5: zoeal larval stage 1-5; ME: megalopa; JU: juvenile crab. (B) The expression of SpCTL6 in molting process of juvenile crabs was analyzed by relative qPCR ( $n=5)$; The expression pattern of SpCTL6 in Z1 ( $\mathrm{n}=5$ ) and megalopa $(n=5)$ after LPS (C, D) and $V$. alginolyticus $(\mathbf{E}, \mathbf{F})$ challenges was analyzed by relative qPCR. Significant difference was indicated by asterisks as ${ }^{*} p<0.05$.

of rSpCTL6 (from $1.5 \mu \mathrm{M}$ to $48 \mu \mathrm{M}$ ) had no cytotoxic effect on the primarily cultured crab hemocytes (Figure 6A), which would provide evidence for the safety use of this protein in subsequent in vivo study.

In addition, it was found that low concentration rSpCTL6 treatment $(10.5 \mu \mathrm{M})$ could significantly reduce the endotoxin level of $V$. alginolyticus, which also showed a dose-dependent manner, that is, higher protein treatment concentration, lower bacterial endotoxin level (Figure 6B). And under the treatment of $84 \mu \mathrm{M}$ rSpCTL6, the endotoxin level was reduced by about $70 \%$ (Figure 6B).

\section{The Immunoprotective Effect of rSpCTL6 on Mud Crab S. Paramamosain Challenged with V. Alginolyticus}

In order to investigate the immunoprotective effect of rSpCTL6 and its regulatory role on the immune system of mud crab $S$. paramamosain under bacterial infection, a series of assays were conducted, including mortality test, bacterial load assay, enzymatic activity assay, and immune-related genes expression analysis.

The schematic diagram of the bacterial challenge and treatment process was shown in Figure 6C. The mud crab mortality curve was drawn (Figure 6C). The results showed 


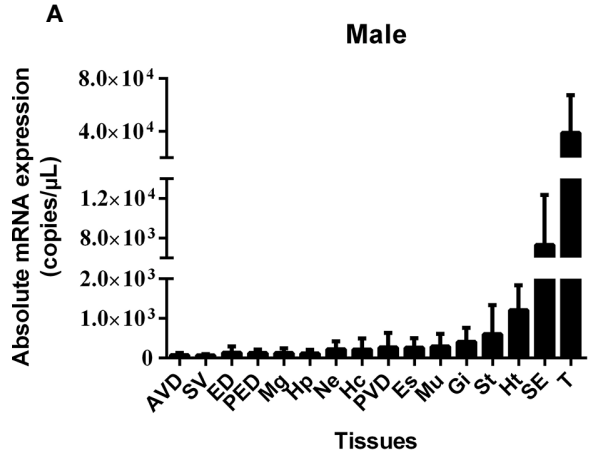

C

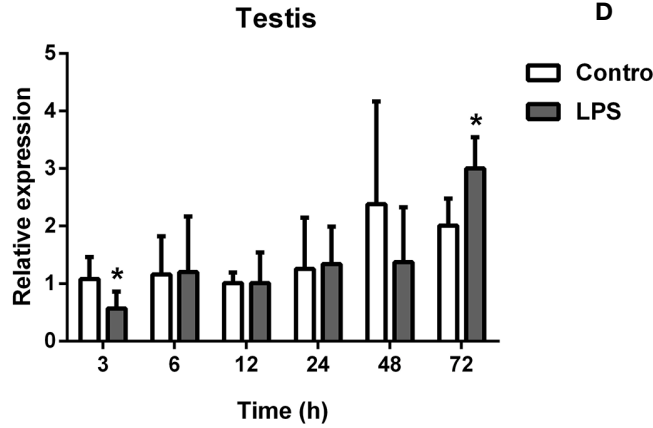

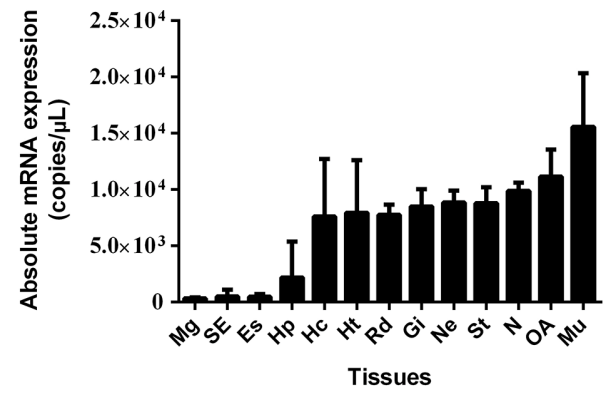

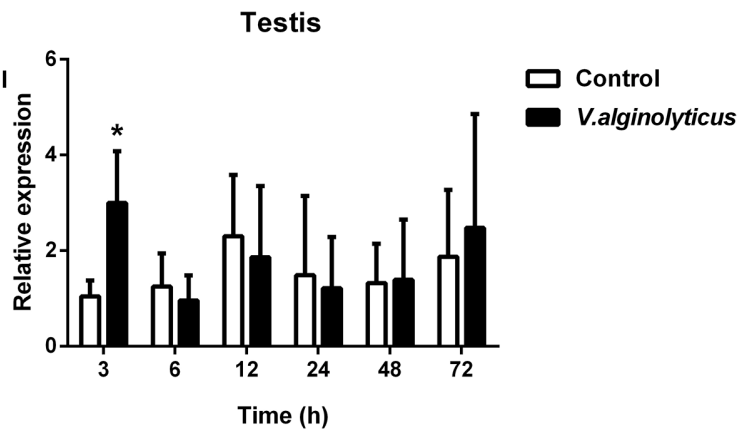

FIGURE 3 | Expression profiles of SpCTL6 gene in S. paramamosain adult. Tissue distribution of SpCTL6 in male (A) and female (B) crabs ( $n=5)$. The expression pattern of SpCTL6 in male testis after LPS (C) and V. alginolyticus (D) challenges $(n=5)$. Significant difference was indicated by asterisks as ${ }^{*} p<0.05$. AVD, anterior vas deferens; SV, seminal vesicle; ED, ejaculatory duct; PED, posterior ejaculatory duct; Mg, midgut; Hp, hepatopancreas; Ne, thoracic ganglion; Hc, hemocytes; PVD, posterior vas deferens; Es, eye stalk; Mu, muscle; Gi, gills; St, stomach; Ht, heart; SE, subcuticular; T, testis; RD, reproductive duct; N: spermathecae; OA, ovaries.

that in the crab saline group, the survival rate dropped sharply in the early stage, and reached about $54 \%$ survival rate after $24 \mathrm{~h}$ of treatment. While for the rSpCTL6 treatment groups $(10 \mu \mathrm{g}$ $\mathrm{crab}^{-1}$ or $\left.20 \mu \mathrm{g} \mathrm{crab}^{-1}\right)$, the survival rate at $24 \mathrm{~h}$ was $80 \%$ and $85.7 \%$, respectively $(P<0.0001)$ (Figure 6C). $120 \mathrm{~h}$ after treatment, the survival rate of the crab saline group was only $11 \%$, while $42.8 \%$ for the $10 \mu \mathrm{g} \mathrm{rSpCTL} 6 \mathrm{crab}^{-1}$ treatment group and $62.9 \%$ for the $20 \mu \mathrm{g}$ rSpCTL6 $\mathrm{crab}^{-1}$ treatment group $(P<0.0001)$ (Figure 6C). These results indicated that rSpCTL6 treatment could significantly increase the survival of mud crab $S$. paramamosain infected with $V$. alginolyticus.

The bacterial loads in gills and hepatopancreas of $S$. paramamosain after bacterial infection and different treatments were determined. As shown in Figure 6D, rSpCTL6 treatment at different time points $(3 \mathrm{~h}, 6 \mathrm{~h}$, and $24 \mathrm{~h})$ could significantly reduce the bacterial number in gills and hepatopancreas of crabs. For example, $3 \mathrm{~h}$ after treatment, the bacterial counts in gills and hepatopancreas of the control group were around $1.19 \times 10^{4} \mathrm{CFU}$ and $1.58 \times 10^{4} \mathrm{CFU}$, while the corresponding bacterial counts in rSpCTL6 treatment group decreased to $1.32 \times 10^{3} \mathrm{CFU}, 1.53 \times 10^{3}$ $\mathrm{CFU}$, respectively. With the treatment time increased (from $3 \mathrm{~h}$ to $24 \mathrm{~h}$ ), the amount of bacteria gradually decreased, no matter in the control group or the treatment group (Figure 6D).

\section{The Regulatory Role of rSpCTL6 on Mud Crab S. Paramamosain Infected by $V$. Alginolyticus}

In order to further investigate the molecular mechanism of the immunoprotective effect of rSpCTL6, we analyzed the activity of several immunological parameters and the expression of genes involved in mud crab immunity. The results showed that the activity of two antioxidant enzymes SOD and POD was significantly upregulated at $3 \mathrm{~h}$ and $24 \mathrm{~h}$ after rSpCTL6 treatment, respectively (Figure 7A). For ACP and AKP, their response time was also different, whose activity was remarkably increased at $6 \mathrm{~h}$ and $24 \mathrm{~h}$ after rSpCTL6 treatment, respectively (Figure 7A). In addition, both LZM and PO activity were found to have significant enhancement at two time points $(3 \mathrm{~h}$ and $24 \mathrm{~h}$ after rSpCTL6 treatment) (Figure 7A).

We randomly chose several typical immune-related genes to analyze their gene expression modulations in hemocytes and hepatopancreas of $S$. paramamosain after rSpCTL6 treatment. The results showed that among them, three genes (including SpSOD, SpCrustin3 and SpALF6) were significantly upregulated in both hemocytes and hepatopancreas at $3 \mathrm{~h}$ after rSpCTL6 treatment, with SpCrustin3 upregulated at $24 \mathrm{~h}$ in hepatopancreas (Figure 7B). While the expression of other 
A

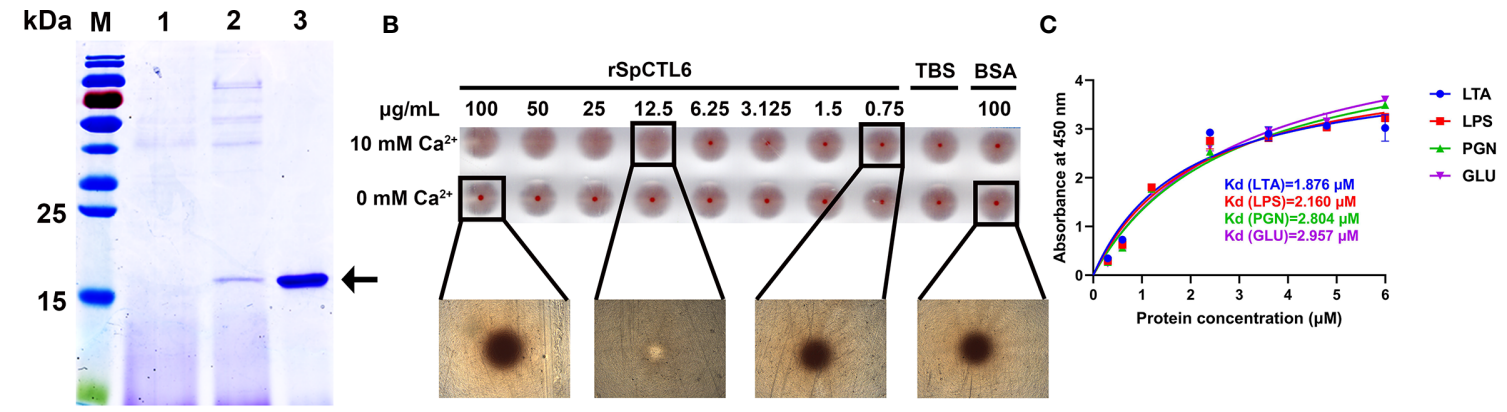

D

G-

$\frac{\mathbf{G +}}{\text { S. aureus }}$
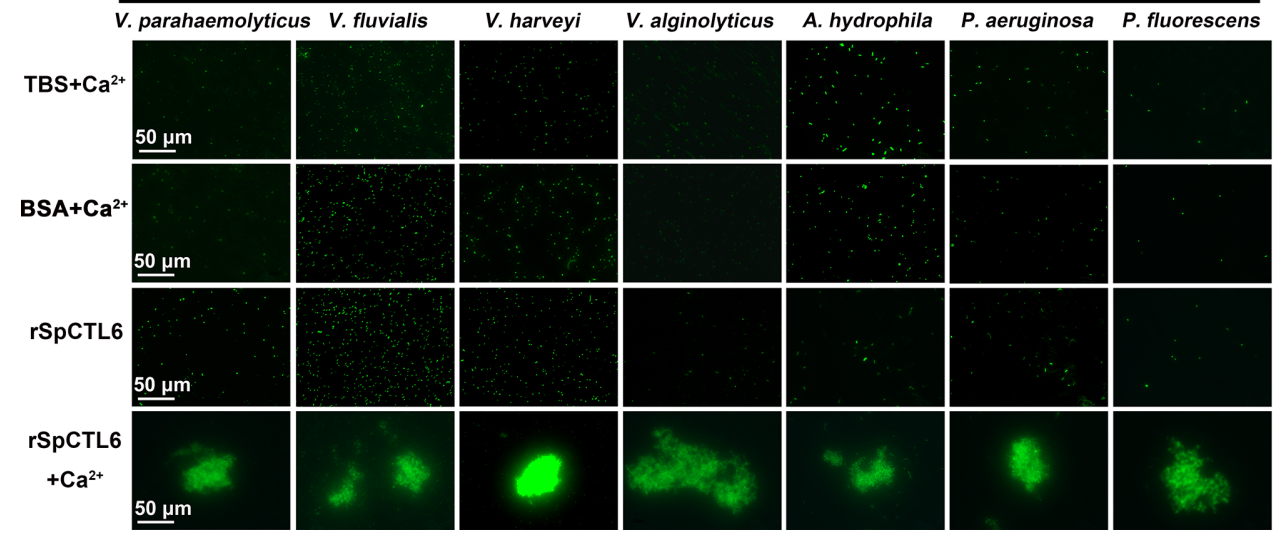

FIGURE 4 | Analysis of hemagglutination, agglutination and binding activity of rSpCTL6. (A) Expression and purification of recombinant SpCTL6. Lane M: protein molecular standard; lane 1: negative control for rSpCTL6 without methanol induction; lane 2: expression of rSpCTL6 after methanol induction; lane 3: purified rSpCTL6. (B) Agglutination activity of rSpCTL6 on mouse erythrocytes. (C) Binding activity of rSpCTL6 to PAMPs (LTA for lipoteichoic acid, LPS for lipopolysaccharide, PGN for peptidoglycan, GLU for glucan). (D) Bacterial agglutinating activity of rSpCTL6 on FITC-labeled Gram-negative bacteria (G-) V. parahaemolyticus, V. fluvialis, V. harveyi, V. alginolyticus, A hydrophila, P. aeruginosa, $P$. fluorescens, and Gram-positive bacteria (G+) S. aureus. The concentration of $\mathrm{CaCl}_{2}$ was $10 \mathrm{mM}$. TBS and BSA were used as control groups.

A

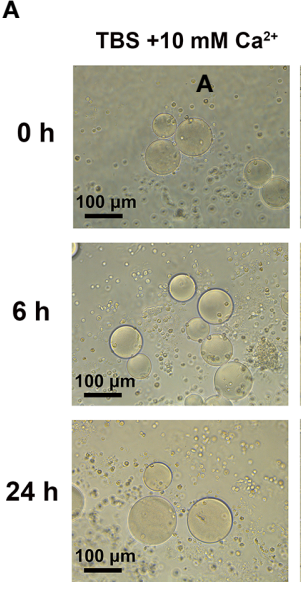

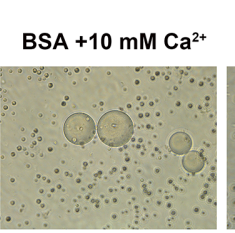
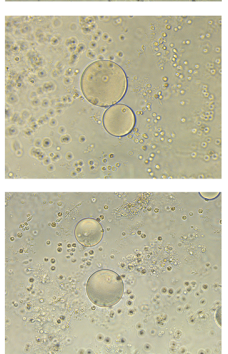

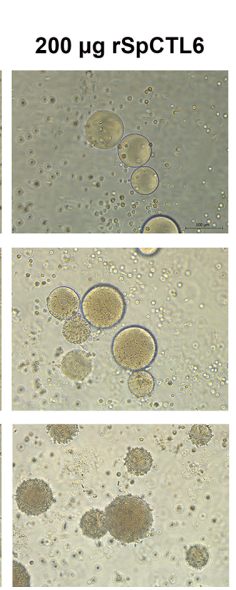

$200 \mu \mathrm{g}$ rSpCTL6 $+10 \mathrm{mM} \mathrm{Ca}^{2+}$
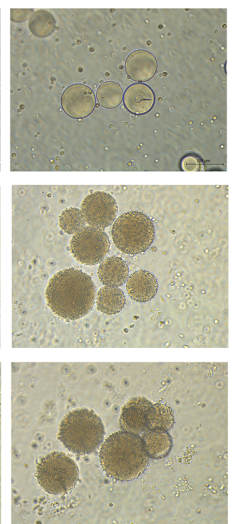

B

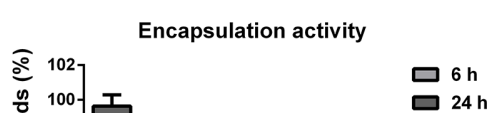

FIGURE 5 | Analysis of encapsulation activity of rSpCTL6. The agrose beads pre-coated with rSpCTL6 were observed for encapsulation by crab hemocytes at 0, 6 and $24 \mathrm{~h}$ post-incubation (A). The beads coated with TBS and BSA were used as control groups. (B) The percentage of beads encapsulated by hemocytes after $6 \mathrm{~h}$ and $24 \mathrm{~h}$ of incubation. 
A

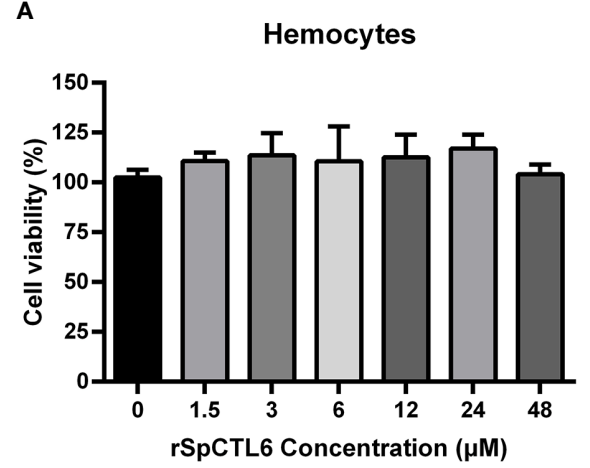

B

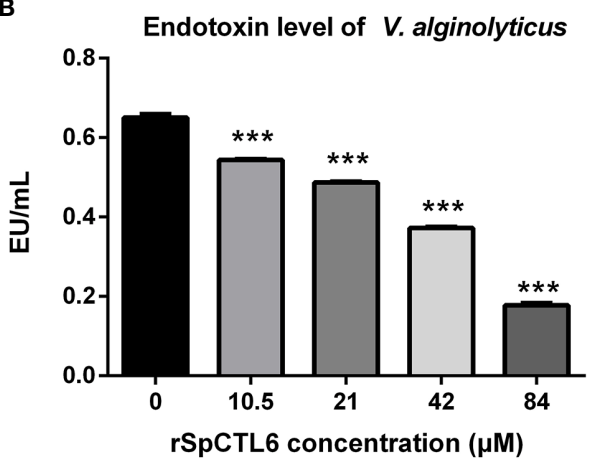

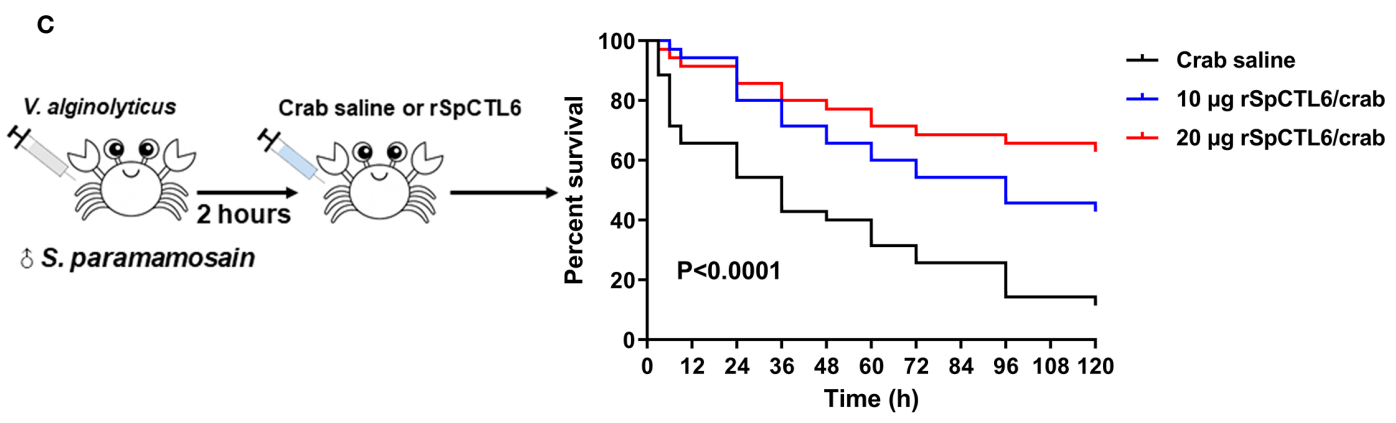

D

Gills

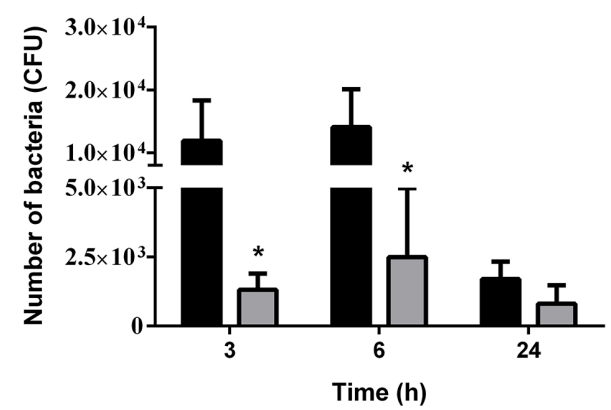

Hepatopancreas

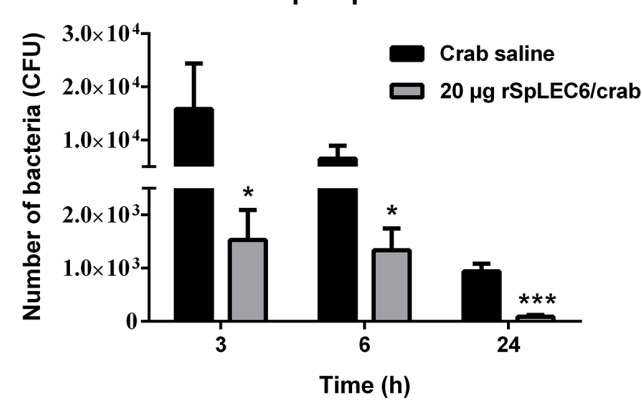

FIGURE 6 | Evaluation of cytotoxicity and endotoxin level of $V$. alginolyticus after rSpCTL6 treatment in vitro and the immunoprotective effect of rSpCTL6 in S. paramamosain. Cytotoxic effect of rSpCTL6 on crab hemocytes (A) and endotoxin level of $V$. alginolyticus after rSpCTL6 treatment in vitro (B). The survival curve of mud crabs after rSpCTL6 treatment (C). Male crabs were challenged with $V$. alginolyticus, and SpCTL6 was injected (10 $\mu \mathrm{g} \mathrm{crab}{ }^{-1} \mathrm{or}^{2} 2 \mu \mathrm{g}$ crab ${ }^{-1}$ ) at $2 \mathrm{~h} \mathrm{post}$ bacterial challenge $(n=35)$. Bacterial clearance of $V$. alginolyticus in the gills and hepatopancreas $(\mathbf{D})$. The bacterial burden in the gills and hepatopancreas were determined at 3, 6 and 24 hpi. * represented $p<0.05$ and ${ }^{* \star \star}$ represented $p<0.001$.

selected genes showed no changes, except for SpALF2 downregulated at $24 \mathrm{~h}$ in hemocytes (Figure S3).

\section{DISCUSSION}

Although the genome of $S$. paramamosain is not available, cDNA library construction and transcriptome analysis has become a powerful tool to identify functional genes $(35,42-44)$. Since the research on SpCTLs is still lack, we screened a new CTL homolog, named SpCTL6 from the embryonic and larval developmental transcriptome database established in our laboratory. It was found that the transcription level of this newly selected CTL gene was relatively high in the entire Zoeal larval stages, and gradually decreased when it developed into megalopa stage and juvenile crabs, which attracted our attention to investigate its function. Subsequent verification by qPCR also confirmed the reliability of the transcriptome data, indicating that SpCTL6 might play important roles in the development of zoeal larval stages. We then cloned the full-length cDNA of SpCTL6 and determined its expression profiles. In addition, we obtained the recombinant protein (rSpCTL6) from eukaryotic 

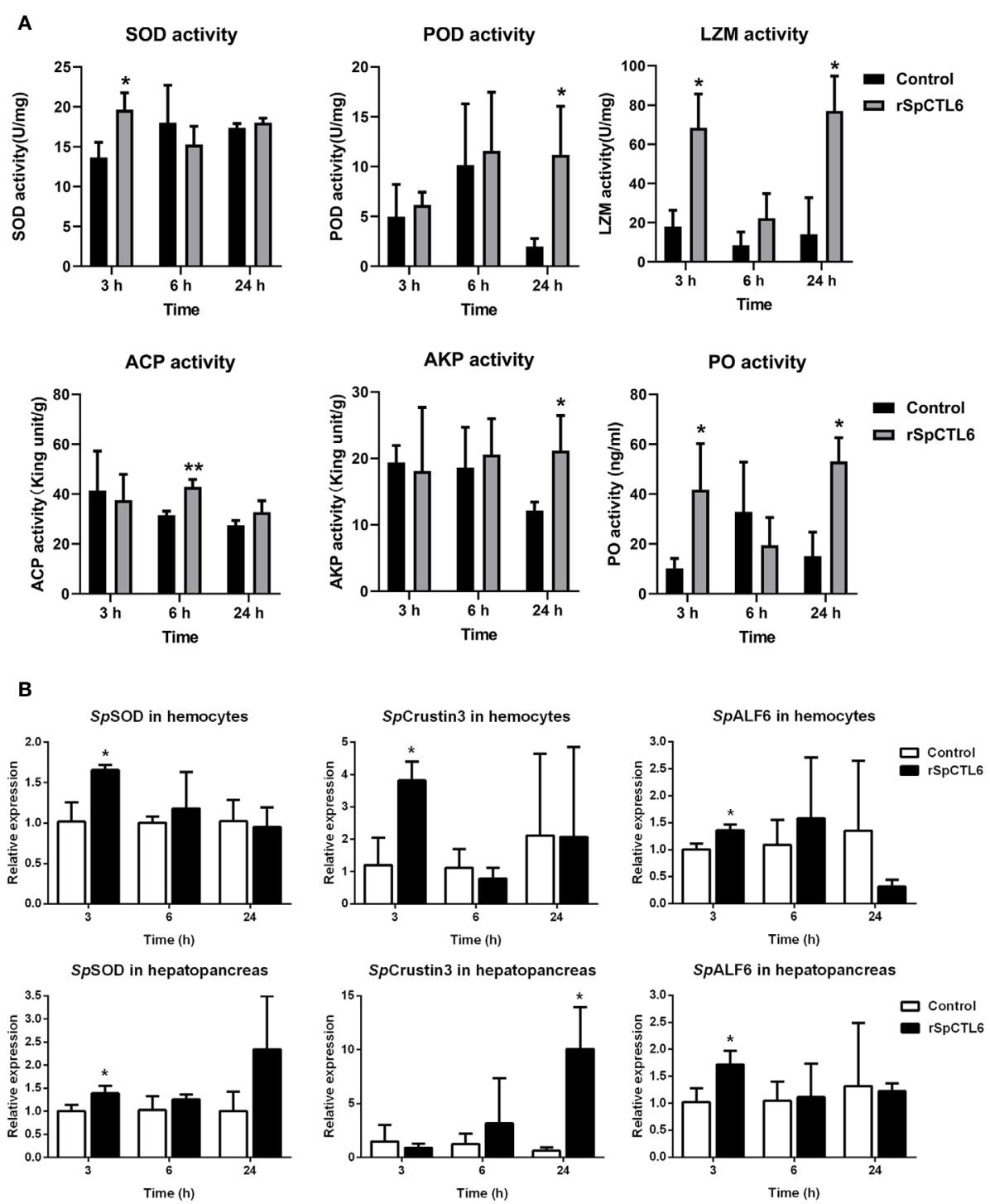

FIGURE 7 | The regulatory role of rSpCTL6 in S. paramamosain. Enzymatic activity analysis (SOD, POD, LZM, ACP, AKP, and PO) in hepatopancreas after rSpCTL6 treatment of $S$. paramamosain challenged with $V$. alginolyticus $(n=3)(\mathbf{A})$. The immune-related gene $(S p S O D, S p C r u s t i n 3$ and $S p A L F 6)$ expression in hemocytes and hepatopancreas were examined $(n=3)(B)$. Significant difference between $r S p C T L 6$ treatment group and the crab saline injection group was indicated by asterisks as ${ }^{*} p<0.05$.

expression system and the immune-related function of rSpCTL6 in vitro and in vivo were elucidated.

Most reported crustacean CTLs have signal peptides, as well as SpCTL6 in this study, indicating that they might belong to secreted expression proteins and act as PRRs, opsonins or effectors to help eliminate invading pathogens (10). In vertebrates, CTL has 4 highly conserved cysteines (Cys), which form disulfide bridges to stabilize its double-loop tertiary structure with $2 \alpha$-helices and $5 \beta$-sheets (11). Besides, there are $4 \mathrm{Ca}^{2+}$-binding sites and site 2 has been confirmed to participate in carbohydrate binding through two typical motifs [EPN (Glu-Pro-Asn) or QPD (Gln-Pro-Asp), and WND (TrpAsn- Asp)] (11). However, since there is no report on crystal structure analysis of crustacean CTLs, we could only obtain information from their structural prediction and sequence alignment with other CTLs. It is found that crustacean CTLs are diverse, in which those conserved motifs in vertebrate are usually mutated, such as EPK (Glu-Pro-Lys), EPS (Glu-Pro-Ser), EPQ (Glu -Pro-Gln), QPG (Gln-Pro-Gly), QPS (Gln-Pro-Ser), QPN (Gln-Pro-Asn), QPT (Gln-Pro-Thr), as summarized in detail in shrimp CTLs (15). Among SpCTLs, only Sp-lectin4 has a typical QPD motif (32), while SpCTL-B contains EPD (GluPro-Asp) motif (33), which is not found in SpLec1, SpLec2, Splectin3 and SpCTL5 $(31,32,34)$. For SpCTL6 in the study, although its amino acid identity is relatively low compared with other crustacean CTLs, it had typical CTL structural 
characteristics, such as a single CTLD, 4 conserved Cys, similar tertiary structure to that of vertebrate CTLs and the mutated $\mathrm{Ca}^{2+}$ binding motif (QPT), clustering into the same branch as the crustacean CTLs, which provided evidence that SpCTL6 is a novel CTL homolog.

In recent years, a large number of studies have focused on the immune function of CTLs, but the role of CTLs in development cannot be ignored. At present, relevant researches have been carried out on insects. It was reported that a CTL HaCTL3 from the cotton bollworm Helicoverpa armigera played an important role in larval growth and development through modulating ecdysone and juvenile hormone signaling (45). Knock-down the expression of Tcctl5 led to developmental defects of Tribolium castaneum, such as reduced survival rate in early adults, and loss of locomotor activity in adults (46). The schlaff (slf) gene coding a putative C-type lectin was necessary for the adhesion between the horizontal cuticle layers of Drosophila (47). So far, research on the function of crustacean CTLs in the development are few and not in-depth. In S. paramamosain, it was found that SpLec1 and SpLec2 had certain expression in the zoeal stages of S. paramamosain, while Sp-lectin3 and Sp-lectin4 were widely distributed in embryos, larvae and crablet stages (31, 32). V. parahaemolyticus challenge could up-regulated the expression of SpLec1 and SpLec2 in megalopa stage of $S$. paramamosain, indicating their role in development and immune response (31). In the study, we systematically analyzed the expression of SpCTL6 in the entire embryonic (Em1-5) and larval stages (Z1-Z5, megalopa) and three stages of juvenile crabs (JU1-3). The absolute copy number of SpCTL6 during zoeal stages reached $0.7 \sim 1 \times 10^{6}$ copies/ $\mu \mathrm{L}$. Such a high transcription level indicated that SpCTL6 might be involved in the modulation of zoeal stage development. However, whether the development-related signaling pathways (such as ecdysone and juvenile hormone signaling) could also be modulated by SpCTL6 similar to HaCTL3 requires further investigation. The upregulated expression pattern of SpCTL6 in Z1 and megalopa stages under immune challenges further demonstrated that SpCTL6 might exert dual roles (developmental modulation and immune response) in the early development of $S$. paramamosain. In addition, during the molting process of juvenile crabs, the expression SpCTL6 was significantly upregulated after molting (JU1 developed to JU2, and JU2 developed to JU3). Since crabs are vulnerable to infection by foreign microorganisms during molting, they might adopt certain strategies, such as increasing the expression of immune factors (including SpCTL6) to resist pathogens. Though the underlying molecular mechanism is still unclear, the results obtained in this study provide new insights into the role of crustacean CTLs in development.

The currently reported crustacean CTLs are widely distributed in various tissues, most of which are predominantly expressed in the hepatopancreas, and some CTLs mainly distributed in hemocytes and gills, or other tissues (heart, gills, intestine, stomach, brain) $(15,21,30,33,34)$. However, there are few reports on the specific high expression of CTLs in the gonads of crustaceans. Interestingly, SpCTL6 was highly expressed in the testis of adult male crabs (its absolute copy number was the highest compared with other male tissues and all female tissues), which is quite different from reported crustacean CTLs. In other aquatic invertebrates, it was found that two CTLs, AjCTL-1 from sea cucumber Apostichopus japonicus and AiCTL-9 from scallop Argopecten irradians also had the highest expression level in gonad $(48,49)$. Wang et al. performed a genome-wide survey on the lectin domain containing proteins (LDCPs) of oyster Crassostrea gigas and found that C-type lectin domain containing proteins (CTLPs) were the most abundant proteins (50). Among them, 40 CTLPs were highly expressed in male gonad, indicating their important role in the reproductive immunity (50). Similar screening methods can also be used in the discovery of crustacean CTLs, which would help us further understand the function of this superfamily. Due to the lack of model animals, it is relatively difficult to investigate the molecular mechanism of functional genes in aquatic invertebrates. In the model organism Caenorhabditis elegans, a CTLP IRG-7 was demonstrated to regulate the immune response of reproductive systems of $C$. elegans to defense against pathogens (51). In S. paramomosain, several male-specific immune effectors antimicrobial peptides (AMPs) have been identified in our previous studies, including scygonadin (5254), SCY2 (55), and scyreprocin (39). All of them have been revealed to participate in the reproductive immunity of mud crabs. In the present study, SpCTL6 was found to be significantly regulated in the testis under LPS or $V$. alginolyticus challenge, indicating that it participated in the immune response of $S$. paramamosain. Therefore, we speculated that the high expression of SpCTL6 in male gonad might also be involved in mud crab reproductive immunity, which will be further studied in the future.

Most functional studies of crustacean CTLs have been conducted through obtaining the recombinant proteins and analyzing the in vitro activity (including antimicrobial, binding, agglutination, and encapsulation activities etc.) (10, 15). Prokaryotic expression system is widely used in the recombinant expression of crustacean CTL proteins, and the vectors included pET-32a, pET-21a, and pGEX-4T-1 etc. $(33,34$, 56). As we all know, the yeast eukaryotic expression system is also a commonly used protein expression system, which can undergo mRNA splicing and post-translational modifications for eukaryotic organisms, thereby would better mimic the existing form of the protein in vivo (57). In particular, the correct spatial structure and modification are very important for protein function. In this study, we obtained high-purity recombinant SpCTL6 (rSpCTL6) from P. pastoris eukaryotic expression system, and confirmed it using mass spectrometry, which provides a critical guarantee for our further functional study.

As PRRs, crustacean CTLs exhibited strong binding activity with pathogen associated molecular patterns (PAMPs, such as LPS, LTA, PGN and GLU) and bacteria, some of which had direct bactericidal activity. In swimming crab $P$. trituberculatus and Chinese mitten crab E. sinensis, several CTLs showed both binding activity with PAMPs and bacteria, such as PtCLec1, PtCTL-2, PtCTL-3, EsCTL1, EsCTL2, and EsCTLDcp (22, 23, 
27, 30). SpCTL-B in S. paramamosain could bind to a variety of bacteria, including Gram-positive bacteria $S$. aureus and Streptococcus hemolyticus, Gram-negative bacteria E. coli, V. parahaemolyticus, $V$. alginolyticus, A. hydrophila and fungi Saccharomyces cerevisiae (33). Several crab CTLs showed potent antibacterial activity, such as SpCTL-B (33), PtCLec1 (30), rEsLecH (21), EsLecA and EsLecG (25). For rSpCTL6, it had binding activity with 4 PAMPs 9LPS, LTA, PGN, and GLU) with moderate binding affinity ["moderate" was defined as $\mathrm{Kd}$ value of $100 \mathrm{nM}$ to $10 \mu \mathrm{M}$ ) (58)]. However, rSpCTL6 could not bind to bacteria and had no obvious antimicrobial activity (data not shown), indicating that it might exert immune functions through other molecular mechanisms.

Agglutination activity is also the hallmark biological function of CTLs. By recognizing molecules on the surface of bacteria, CTLs can directly immobilize pathogens and agglutinate them, so that pathogens will not spread in the body. A large number of studies have shown that most CTLs rely on calcium ions to exert or enhance their agglutination activity (10). Crab CTLs exhibited a broad spectrum of bacterial agglutination activity in $\mathrm{Ca}^{2+}$ dependent manner, especially on several typical aquaculture pathogens, such as $V$. alginolyticus, $V$. parahaemolyticus, $V$. vulnificus, and $A$. hydrophila $(22,23,27,30,33)$. Among them, EsLecA, EsLecG and EsLecD agglutinated E. coli and P. pastoris without relying on calcium ions, and the addition of $\mathrm{Ca}^{2+}$ could significantly enhance their agglutinating activity $(25,59)$. In addition, PtCLec1, PtCTL4 and PtCTL1 had hemagglutination activity on rabbit erythrocytes in the presence of $\mathrm{Ca}^{2+}(28-30)$. Similar broad spectrum bacterial agglutination activity was also found in rSpCTL6, which could agglutinate 5 common pathogens in aquaculture ( $A$. hydrophila, $V$. harveyi, $V$. fluvialis, $V$. parahaemolyticus, and $V$. alginolyticus), and rSpCTL6 had hemagglutination activity on mouse erythrocytes in a $\mathrm{Ca}^{2+}$-dependent manner.

When some large pathogens such as parasites invade the crustacean, the host hemocytes will wrap around them, forming a multiple cell layers, so that those pathogens cannot move freely in the host, and then they will be killed by melanin or endotoxin released by hemocytes (60). Therefore, hemocyte encapsulation ability play an important role in cellular immunity of invertebrates (61). Studies have found that crustacean CTLs can enhance the ability of hemocytes to encapsulate large pathogens and then followed by melanization (15). Agarose beads are usually used as invading objects to evaluate the effect of CTLs on the encapsulation activity of hemocytes. When the agarose beads were incubated with PtCTL-2 and PtCTL-3 from P. trituberculatus separately, and then the hemocytes were added, the hemocytes encapsulated $57 \%$ and $45 \%$ of agarose beads respectively, while the control group had no obvious coating effect (27). In E. sinensis, EsLecA, EsLecG, EsLecD and EsLecF could also activate the encapsulation activity of hemocytes on agarose beads $(25,59,62)$. The results showed that after $24 \mathrm{~h}$ of incubation, almost 100\% of EsLecD-coated agarose beads were encapsulated by hemocytes, which might be due to the high binding affinity of EsLecD to hemocyte surface molecules (59). Similarly, in our study, rSpCTL6 significantly enhanced the hemocyte encapsulation on agarose beads and might cause hemocyte melanization to help eliminate invading pathogens, indicating its important role in cellular immunity of S. paramamosain.

Research on the function of recombinant CTLs in vitro is far from enough, and elucidating the immune protective and regulatory effects of CTL proteins in vivo will provide important evidences for further understanding their immune functions. Most of the reported crustacean CTLs conducted the in vivo bacterial clearance assay to evaluate the in vivo effects of recombinant CTLs. They first pre-incubated the bacteria and recombinant CTL protein for a period of time, and then coinjected them into animals, and finally counted the numbers of living bacteria in the hemolymph. The results showed that this treatment could remarkably accelerate the bacteria clearance, such as rPtCLec1 (30), EsCTL1 and EsCTL2 (22), EsCTLDcp (23) in crab, PcLec3 (63), PcLec4 (64), MnCTL (56), MrCTL (65), LvCTL3 (66), FmLC4 (67), and FcLec4 (68) in crayfish, prawn and shrimp. Whether CTLs can significantly improve the survival rate of animals under pathogenic infection is an important evidence to clarify their immune protective effects. PcLec4 overexpression in vivo exhibited obviously higher survival rate $(50 \%$ at the ninth day) than that of the control groups (10\% at the ninth day) (64). Pre-incubated rPcLT and WSSV could effectively protect crayfish Procambarus clarkii from WSSV infection, with a much higher survival rate (around 75\%) than that of the control groups (3-5\%) (69). Injection of WSSV or $V$. alginolyticus together with rLvCTL3 could significantly reduce the mortality of $L$. vannamei infected by WSSV and $V$. alginolyticus (66). In our study, the treatment method was different from those reports mentioned above. We first infected the crabs with $V$. alginolyticus and 2 hours later, injected rSpCTL6 or crab saline into the crabs. We would like to know the in vivo protective effect of rSpCTL6 under pathogenic infection. The results showed that rSpCTL6 treatment could also enhance the bacterial clearance in gills and hepatopancreas. In addition, rSpCTL6 treatment could greatly improve the survival of infected crabs [increased by about 50\% (62.9\% in rSpCTL6 treatment group, $11 \%$ in the control group in $120 \mathrm{~h}$ )], which would provide a valuable information for the disease control in mud crab aquaculture.

At present, the regulatory role of crustacean CTLs in the immune system has been extensively studied mainly through the use of RNA interference (RNAi), which is a powerful tool for revealing the molecular mechanism of functional genes (15). In crabs, such as in E. sinensis, RNAi of EsLecH could down-regulate the expression of several AMPs (including Es-DWD1, EsALF, and EsALF-2) and phosphorylation of JNK (21). When the mRNA level of JNK was inhibited, the addition of rEsLecH could not cause changes in AMP expression, indicating that EsLecH required JNK signaling to perform its regulation function (21). Knock down of PtCLec1 from $P$. trituberculatus could regulate the expression of genes involved in phagocytosis, proPO system, complement system, Toll and IMD pathways, and AMPs, indicating its key regulatory role in the innate immunity of $P$. trituberculatus (30). In rPcLT treatment shrimp, PO and SOD activity increased significantly at different time points (69). In S. paramamosain, 
several AMPs (SpHistin, SpALF4, SpALF5 and SpALF6), spSTAT and hemocyte phagocytosis were all regulated by SpCTL-B (also evaluated through RNAi), suggesting that SpCTL-B played important roles both in the humoral and cellular immunity of mud crabs (33). In order to understand the underlying molecular mechanism of the immunoprotective effect of SpCTL6, we further analyzed the expression of immune-related genes and several immunological parameters after rSpCTL6 treatment. We found that the expression of two AMP genes SpCrustin3 and SpALF6, an antioxidant gene SOD, and all the tested parameters (including SOD, POD, LZM, ACP, AKP, and PO activity) had been upregulated, indicating the regulatory role of rSpALF6 in the innate immunity of $S$. paramamosain. However, the underlying regulatory mechanism of CTLs is complicated and we are still far from understanding it. As shown in a previous study, it was found that the silencing of LvLdlrCTL (a CTL containing a low-density lipoprotein receptor (LDLR) class A domain) from L. vannamei down-regulated the expression of 3 AMPs (crustin, ALF3, and PEN3), but 2 other AMPs (PEN2 and PEN4) were upregulated (70). Similarly, in our study, 2 AMPs (SpCrustin3 and SpALF6) were upregulated at $3 \mathrm{~h}$ in the hemocytes, while another AMP SpALF2 was down-regulated at $24 \mathrm{~h}$ in the hemocytes after rSpCTL6 treatment. In the early stage $(3 \mathrm{~h}$ and $6 \mathrm{~h}$ after rSpCTL6 treatment), the bacterial load in crabs was still high (Figure 6D), which might require increased enzyme activity and expression of AMPs to resist bacteria. Our results corresponded to this hypothesis, for example, the activities of a variety of immunological parameters in the hepatopancreas (including SOD, LZM, ACP and PO) and several immune-related genes in hemocytes (including SpSOD, SpCrustin3, SpALF6) were upregulated at $3 \mathrm{~h}$ or $6 \mathrm{~h}$ after rSpCTL6 treatment, suggesting that rSpCTL6 might activate the innate immune system of $S$. paramamosain, thereby quickly eliminating the invading bacteria. And at the $24 \mathrm{~h}$ time point, the number of bacteria decreased to a low level, we speculated that crabs need to adopted anti-inflammatory strategies to help the body recover. Therefore, the downregulation of SpALF2 might be due to the need for anti-inflammatory response and the maintenance of homeostasis, which requires further indepth investigation.

In summary, this study characterized a new CTL homolog SpCTL6 from mud crab S. paramamosain. SpCTL6 was highly expressed in the entire zoeal larval stages and widely distributed in adult crab tissues with the highest transcription level in testis. It could be significantly upregulated in two larval stages (Z1 and megalopa stages) and adult crab testis under immune challenges. During the molting process of juvenile crabs, the expression level of SpCTL6 was remarkably increased after molting. Recombinant SpCTL6 was successfully obtained from eukaryotic expression system. rSpCTL6 exhibited binding activity with PAMPs and had a broad spectrum of bacterial agglutination activity in a $\mathrm{Ca}^{2}$ ${ }^{+}$-dependent manner. rSpCTL6 could enhance the encapsulation activity of hemocytes and it had no cytotoxic effect on hemocytes. Furthermore, rSpCTL6 treatment could significantly reduce the endotoxin level of $V$. alginolyticus in vitro and greatly improved the survival of $S$. paramamosain under bacterial infection. The immunoprotective effect of rSpCTL6 might be due to the regulatory role of rSpALF6 in the immune-related genes and immunological parameters. Our study provides important information for understanding the immune defense of mud crabs and would facilitate the development of effective strategies for mud crab aquaculture disease control.

\section{DATA AVAILABILITY STATEMENT}

The original contributions presented in the study are included in the article/Supplementary Material. Further inquiries can be directed to the corresponding author.

\section{ETHICS STATEMENT}

The animal study was reviewed and approved by the Laboratory Animal Management and Ethics Committee of Xiamen University.

\section{AUTHOR CONTRIBUTIONS}

WQ: Data curation, formal analysis, investigation, and methodology. RC, SL, XZ, and MX: Investigation and methodology. FC: Conceptualization, funding acquisition, project administration, supervision; writing-original draft, and writing-review and editing. KW: Funding acquisition, project administration, and writing-review and editing. All authors contributed to the article and approved the submitted version.

\section{FUNDING}

This study was supported by grants (grant \# U1805233/ \# 41806162) from the National Natural Science Foundation of China (NSFC), the Fundamental Research Funds from Central Universities (grant \# 20720190109), the Fujian Marine Economic Development 395 Subsidy Fund Project (Grant \# FJHJF-L-2019-1) from the Fujian Ocean and Fisheries Department and a grant (\# 3502Z20203012) from the Xiamen Science and Technology Planning Project.

\section{ACKNOWLEDGMENTS}

We thank laboratory engineers Hui Peng and Zhiyong Lin for providing technical assistance. We thank Yaying $\mathrm{Wu}$, Zheni Xu and C.C. Xie for mass spectrometry experiments and data analysis.

\section{SUPPLEMENTARY MATERIAL}

The Supplementary Material for this article can be found online at: https://www.frontiersin.org/articles/10.3389/fimmu.2021. 661823/full\#supplementary-material 


\section{REFERENCES}

1. Mirera OD. Trends in exploitation, development and management of artisanal mud crab (Scylla serrata-Forsskål-1775) fishery and small-scale culture in Kenya: An overview. Ocean Coast Manage (2011) 54:844-55. doi: 10.1016/j.ocecoaman.2011.08.001

2. Azra MN, Ikhwanuddin M. A review of maturation diets for mud crab genus Scylla broodstock: Present research, problems and future perspective. Saudi J Biol Sci (2016) 23:257-67. doi: 10.1016/j.sjbs.2015.03.011

3. Hungria DB, Tavares CPD, Pereira LA, da Silva UDT, Ostrensky A. Global status of production and commercialization of soft-shell crabs. Aquacult Int (2017) 25:2213-26. doi: 10.1007/s10499-017-0183-5

4. Ministry of Agriculture and Rural Affairs of the People's Republic of China, Fisheries Administration; National Fisheries Technology Promotion Station, China; Chinese Fisheries Society. China Fishery Statistical Yearbook 2019. China Agriculture Press (2019). pp. 1-152.

5. Hamasaki K, Obata Y, Dan S, Kitada S. A review of seed production and stock enhancement for commercially important portunid crabs in Japan. Aquacult Int (2011) 19:217-35. doi: 10.1007/s10499-010-9387-7

6. Chen F, Wang K. Characterization of the innate immunity in the mud crab Scylla paramamosain. Fish Shellfish Immunol (2019) 93:436-48. doi: 10.1016/ j.fsi.2019.07.076

7. Quinitio ET, de la Cruz JJ, Eguia MRR, Parado-Estepa FD, Pates G, LavillaPitogo CR. Domestication of the mud crab Scylla serrata. Aquacult Int (2011) 19:237-50. doi: 10.1007/s10499-010-9381-0

8. Ye HH, Tao Y, Wang GZ, Lin QW, Chen XL, Li SJ. Experimental nursery culture of the mud crab Scylla paramamosain (Estampador) in China. Aquacult Int (2011) 19:313-21. doi: 10.1007/s10499-010-9399-3

9. Robinson MJ, Sancho D, Slack EC, LeibundGut-Landmann S, Reis e Sousa C. Myeloid C-type lectins in innate immunity. Nat Immunol (2006) 7:1258-65. doi: $10.1038 /$ ni1417

10. Pees B, Yang WT, Zarate-Potes A, Schulenburg H, Dierking K. High innate immune specificity through diversified C-type lectin-like domain proteins in invertebrates. J Innate Immun (2016) 8:129-42. doi: 10.1159/000441475

11. Zelensky AN, Gready JE. The C-type lectin-like domain superfamily. FEBS J (2005) 272:6179-217. doi: 10.1111/j.1742-4658.2005.05031.x

12. Kilpatrick DC, 187-97. Animal lectins: a historical introduction and overview. Biochim Biophys Acta (2002) 1572:187-97. doi: 10.1016/S0304-4165(02) 00308-2

13. van Gisbergen KP, Ludwig IS, Geijtenbeek TB, van Kooyk Y. Interactions of DCSIGN with Mac-1 and CEACAM1 regulate contact between dendritic cells and neutrophils. FEBS Lett (2005) 579:6159-68. doi: 10.1016/j.febslet.2005.09.089

14. Vaishnava S, Yamamoto M, Severson KM, Ruhn KA, Yu X, Koren O, et al. The antibacterial lectin RegIIIgamma promotes the spatial segregation of microbiota and host in the intestine. Science (2011) 334:255-8. doi: 10.1126/science.1209791

15. Wang XW, Wang JX. Diversity and multiple functions of lectins in shrimp immunity. Dev Comp Immunol (2013) 39:27-38. doi: 10.1016/j.dci.2012.04.009

16. Wang XW, Vasta GR, Wang JX. The functional relevance of shrimp C-type lectins in host-pathogen interactions. Dev Comp Immunol (2020) 109:103708. doi: 10.1016/j.dci.2020.103708

17. Zhang XJ, Yuan JB, Sun YM, Li SH, Gao Y, Yu Y, et al. Penaeid shrimp genome provides insights into benthic adaptation and frequent molting. Nat Commun (2019) 10:356. doi: 10.1038/s41467-018-08197-4

18. Gutekunst J, Andriantsoa R, Falckenhayn C, Hanna K, Stein W, Rasamy J, et al. Clonal genome evolution and rapid invasive spread of the marbled crayfish. Nat Ecol Evol (2018) 2:567-73. doi: 10.1038/s41559-018-0467-9

19. Song L, Bian C, Luo Y, Wang L, You X, Li J, et al. Draft genome of the Chinese mitten crab, Eriocheir sinensis. Gigascience (2016) 5:5. doi: 10.1186/s13742016-0112-y

20. Tang B, Zhang D, Li H, Jiang S, Zhang H, Xuan F, et al. Chromosome-level genome assembly reveals the unique genome evolution of the swimming crab (Portunus trituberculatus). Gigascience (2020) 9:1-10. doi: 10.1093/ gigascience/giz161

21. Zhu YT, Zhang X, Wang SC, Li WW, Wang Q. Antimicrobial functions of EsLecH, a C-type lectin, via JNK pathway in the Chinese mitten crab, Eriocheir sinensis. Dev Comp Immunol (2016) 61:225-35. doi: 10.1016/ j.dci.2016.04.007
22. Huang Y, Huang X, Wang Z, Tan JM, Hui KM, Wang W, et al. Function of two novel single-CRD containing C-type lectins in innate immunity from Eriocheir sinensis. Fish Shellfish Immunol (2014) 37:313-21. doi: 10.1016/ j.fsi.2014.02.001

23. Huang Y, An L, Hui KM, Ren Q, Wang W. An LDLa domain-containing Ctype lectin is involved in the innate immunity of Eriocheir sinensis. Dev Comp Immunol (2014) 42:333-44. doi: 10.1016/j.dci.2013.10.004

24. Wang L, Wang L, Zhang D, Li F, Wang M, Huang M, et al. A novel C-type lectin from crab Eriocheir sinensis functions as pattern recognition receptor enhancing cellular encapsulation. Fish Shellfish Immunol (2013) 34:832-42. doi: 10.1016/j.fsi.2012.12.010

25. Jin XK, Li S, Guo XN, Cheng L, Wu MH, Tan SJ, et al. Two antibacterial Ctype lectins from crustacean, Eriocheir sinensis, stimulated cellular encapsulation in vitro. Dev Comp Immunol (2013) 41:544-52. doi: 10.1016/ j.dci.2013.07.016

26. Guo HZ, Zou PF, Fu JP, Guo Z, Zhu BK, Nie P, et al. Characterization of two C-type lectin-like domain (CTLD)-containing proteins from the cDNA library of Chinese mitten crab Eriocheir sinensis. Fish Shellfish Immunol (2011) 30:515-24. doi: 10.1016/j.fsi.2010.11.027

27. Huang $\mathrm{M}, \mathrm{Mu} \mathrm{C}, \mathrm{Wu} \mathrm{Y}$, Ye F, Wang $\mathrm{D}$, Sun $\mathrm{C}$, et al. The functional characterization and comparison of two single CRD containing C-type lectins with novel and typical key motifs from Portunus trituberculatus. Fish Shellfish Immunol (2017) 70:398-407. doi: 10.1016/j.fsi.2017.09.029

28. Lu J, Yu Z, Mu C, Li R, Song W, Wang C. Characterization and functional analysis of a novel C-type lectin from the swimming crab Portunus trituberculatus. Fish Shellfish Immunol (2017) 64:185-92. doi: 10.1016/ j.fsi.2017.03.013

29. Zhang X, Lu J, Mu C, Li R, Song W, Ye Y, et al. Molecular cloning of a C-type lectin from Portunus trituberculatus, which might be involved in the innate immune response. Fish Shellfish Immunol (2018) 76:216-23. doi: 10.1016/ j.fsi.2018.01.051

30. Su Y, Liu Y, Gao F, Cui Z. A novel C-type lectin with a YPD motif from Portunus trituberculatus (PtCLec1) mediating pathogen recognition and opsonization. Dev Comp Immunol (2020) 106:103609. doi: 10.1016/ j.dci.2020.103609

31. Jiang K, Zhang D, Zhang F, Sun M, Qi L, Zhang S, et al. Isolation of the C-type lectin like-domain cDNAs from the mud crab Scylla paramamosain Estampador, 1949, and its expression profiles in various Tissues, during larval development, and under Vibrio challenge. Crustaceana (2012) 85:817-34. doi: 10.1163/156854012X650269

32. Duan LP, Huang B, Zhou LH, Liang Y, Nie P, Huang WS. Molecular cloning, characterization and expression of two novel lectins in mud crab, Scylla paramamosain. Acta Hydrobiol Sin (2015) 39:321-30. doi: 10.7541/2015.43

33. Wei X, Wang L, Sun W, Zhang M, Ma H, Zhang Y, et al. (SpCTL-B) regulates the expression of antimicrobial peptides and promotes phagocytosis in mud crab Scylla paramamosain. Dev Comp Immunol (2018) 84:213-29. doi: 10.1016/j.dci.2018.02.016

34. Zhang W, Zhang Z, Mu C, Li R, Ye Y, Zhang H, et al. Molecular cloning, characterization, and expression of a C-type lectin from Scylla paramamosain, which might be involved in the innate immune response. Fish Shellfish Immunol (2019) 93:251-7. doi: 10.1016/j.fsi.2019.07.035

35. Chen FY, Liu HP, Bo J, Ren HL, Wang KJ. Identification of genes differentially expressed in hemocytes of Scylla paramamosain in response to lipopolysaccharide. Fish Shellfish Immunol (2010) 28:167-77. doi: 10.1016/ j.fsi.2009.10.017

36. Zeng C, Li S, Zeng H. Studies on the morphology of the larvae of Scylla serrata (Forskal). J Zhanjiang Ocean Univ (2001) 2:1-6.

37. Huang S, Li W. Study on the larval development of Scylla serrata (Forskal). J Fisheries China (1965) 4:24-30.

38. Livak KJ, Schmittgen TD. Analysis of relative gene expression data using realtime quantitative PCR and the $2^{\text {-DDCT }}$ Method. Methods (2001) 25:402. doi: 10.1006/meth.2001.1262

39. Yang Y, Chen F, Chen HY, Peng H, Hao H, Wang KJ. A novel antimicrobial peptide scyreprocin from mud crab Scylla paramamosain showing potent antifungal and anti-biofilm activity. Front Microbiol (2020) 11:1589. doi: 10.3389/fmicb.2020.01589

40. Deepika A, Makesh M, Rajendran KV. Development of primary cell cultures from mud crab, Scylla serrata, and their potential as an in vitro model for the 
replication of white spot syndrome virus. In Vitro Cell Dev Biol: Anim (2014) 50:406-16. doi: 10.1007/s11626-013-9718-x

41. Qiao K, Zhang YQ, Wang SP, Zhe AN, Hao H, Chen FY, et al. The optimization of primary hemocyte culture of Scylla paramamosain. China Anim Husbandry Vet Med (2014) 41:145-9.

42. Cheng CH, Ma HL, Deng YQ, Feng J, Jie YK, Guo ZX. Effects of Vibrio parahaemolyticus infection on physiological response, histopathology and transcriptome changes in the mud crab (Scylla paramamosain). Fish Shellfish Immunol (2020) 106:197-204. doi: 10.1016/j.fsi.2020.07.061

43. Liu S, Chen G, Xu H, Zou W, Yan W, Wang Q, et al. Transcriptome analysis of mud crab (Scylla paramamosain) gills in response to Mud crab reovirus (MCRV). Fish Shellfish Immunol (2017) 60:545-53. doi: 10.1016/j.fsi.2016.07.033

44. Xie C, Chen Y, Sun W, Ding J, Zhou L, Wang S, et al. Transcriptome and expression profiling analysis of the hemocytes reveals a large number of immune-related genes in mud crab Scylla paramamosain during Vibrio parahaemolyticus infection. PloS One (2014) 9:e114500. doi: 10.1371/ journal.pone. 0114500

45. Wang W, Wang G, Zhuo X, Liu Y, Tang L, Liu X, et al. C-type lectin-mediated microbial homeostasis is critical for Helicoverpa armigera larval growth and development. PloS Pathog (2020) 16:e1008901. doi: 10.1371/ journal.ppat.1008901

46. Li J, Bi J, Zhang P, Wang Z, Zhong Y, Xu S, et al. Functions of a C-type lectin with a single carbohydrate-recognition domain in the innate immunity and movement of the red flour beetle, Tribolium castaneum. Insect Mol Biol (2021) 30:90-101. doi: 10.1111/imb.12680

47. Zuber R, Shaik KS, Meyer F, Ho HN, Speidel A, Gehring N, et al. The putative C-type lectin Schlaff ensures epidermal barrier compactness in Drosophila. Sci Rep (2019) 9:5374. doi: 10.1038/s41598-019-41734-9

48. Wei X, Liu X, Yang J, Wang S, Sun G, Yang J. Critical roles of sea cucumber Ctype lectin in non-self recognition and bacterial clearance. Fish Shellfish Immunol (2015) 45:791-9. doi: 10.1016/j.fsi.2015.05.037

49. Wang L, Wang L, Yang J, Zhang H, Huang M, Kong P, et al. A multi-CRD Ctype lectin with broad recognition spectrum and cellular adhesion from Argopecten irradians. Dev Comp Immunol (2012) 36:591-601. doi: 10.1016/ j.dci.2011.10.002

50. Wang W, Gong C, Han Z, Lv X, Liu S, Wang L, et al. The lectin domain containing proteins with mucosal immunity and digestive functions in oyster Crassostrea gigas. Fish Shellfish Immunol (2019) 89:237-47. doi: 10.1016/ j.fsi.2019.03.067

51. Yunger E, Safra M, Levi-Ferber M, Haviv-Chesner A, Henis-Korenblit S. Innate immunity mediated longevity and longevity induced by germ cell removal converge on the C-type lectin domain protein IRG-7. PloS Genet (2017) 13:e1006577. doi: 10.1371/journal.pgen.1006577

52. Wang KJ, Huang WS, Yang M, Chen HY, Bo J, Li SJ, et al. A male-specific expression gene, encodes a novel anionic antimicrobial peptide, scygonadin, in Scylla serrata. Mol Immunol (2007) 44:1961-8. doi: 10.1016/j.molimm.2006.09.036

53. Wang KJ, Huang WS, Yang M, Cai JJ. Scygonadin, a novel male-specific anionic antibacterial protein from Scylla serrata (forskål, 1775). FASEB J (2006) 20:A974-4. doi: 10.1096/fasebj.20.5.A974-a

54. Huang WS, Wang KJ, Yang M, Cai JJ, Li SJ, Wang GZ. Purification and part characterization of a novel antibacterial protein Scygonadin, isolated from the seminal plasma of mud crab, Scylla serrata (forskål, 1775). J Exp Mar Biol Ecol (2006) 339:37-42. doi: 10.1016/j.jembe.2006.06.029

55. Qiao K, Xu WF, Chen HY, Peng H, Zhang YQ, Huang WS, et al. A new antimicrobial peptide SCY2 identified in Scylla Paramamosain exerting a potential role of reproductive immunity. Fish Shellfish Immunol (2016) 51:251-62. doi: 10.1016/j.fsi.2016.02.022

56. Huang $\mathrm{X}$, Li T, Jin $\mathrm{M}$, Yin $\mathrm{S}$, Wang $\mathrm{W}$, Ren Q. Identification of a Macrobrachium nipponense C-type lectin with a close evolutionary relationship to vertebrate lectins. Mol Immunol (2017) 87:141-51. doi: 10.1016/j.molimm.2017.04.009

57. Thakur R, Shankar J. Strategies for gene expression in prokaryotic and eukaryotic system. In: VC Kalia, AK Saini, editors. Metabolic Engineering for Bioactive Compounds: Strategies and Processes. Singapore: Springer Singapore (2017). p. 223-47.

58. Watson JD, Baker TA, Bell SP, Gann A, Levine M, Losick R. Molecular Biology of the Gene 5th ed. Benjamin Cummings: Cold Spring Harbor Laboratory Press (1976).

59. Guo XN, Jin XK, Li S, Yu AQ, Wu MH, Tan SJ, et al. A novel C-type lectin from Eriocheir sinensis functions as a pattern recognition receptor with antibacterial activity. Fish Shellfish Immunol (2013) 35:1554-65. doi: 10.1016/j.fsi.2013.08.021

60. Cerenius L, Soderhall K. The prophenoloxidase-activating system in invertebrates. Immunol Rev (2004) 198:116-26. doi: 10.1111/j.01052896.2004.00116.x

61. Nappi A, Poirie M, Carton Y. The role of melanization and cytotoxic by-products in the cellular immune responses of Drosophila against parasitic wasps. Adv Parasitol (2009) 70:99-121. doi: 10.1016/S0065-308X (09)70004-1

62. Jin XK, Guo XN, Li S, Wu MH, Zhu YT, Yu AQ, et al. Association of a hepatopancreas-specific C-type lectin with the antibacterial response of Eriocheir sinensis. PloS One (2013) 8:e76132. doi: 10.1371/ journal.pone.0076132

63. Zhang XW, Wang Y, Wang XW, Wang L, Mu Y, Wang JX. A C-type lectin with an immunoglobulin-like domain promotes phagocytosis of hemocytes in crayfish Procambarus clarkii. Sci Rep (2016) 6:29924. doi: 10.1038/ srep29924

64. Zhang XW, Liu YY, Mu Y, Ren Q, Zhao XF, Wang JX. Overexpression of a Ctype lectin enhances bacterial resistance in red swamp crayfish Procambarus clarkii. Fish Shellfish Immunol (2013) 34:1112-8. doi: 10.1016/j.fsi.2013.01.020

65. Huang X, Feng JL, Jin M, Ren Q, Wang W. C-type lectin (MrCTL) from the giant freshwater prawn Macrobrachium rosenbergii participates in innate immunity. Fish Shellfish Immunol (2016) 58:136-44. doi: 10.1016/ j.fsi.2016.08.006

66. Li M, Li C, Ma C, Li H, Zuo H, Weng S, et al. Identification of a C-type lectin with antiviral and antibacterial activity from pacific white shrimp Litopenaeus vannamei. Dev Comp Immunol (2014) 46:231-40. doi: 10.1016/ j.dci.2014.04.014

67. Utarabhand P, Thepnarong S, Runsaeng P. Lipopolysaccharide-specific binding C-type lectin with one CRD domain from Fenneropenaeus merguiensis (FmLC4) functions as a pattern recognition receptor in shrimp innate immunity. Fish Shellfish Immunol (2017) 69:236-46. doi: 10.1016/ j.fsi.2017.08.028

68. Wang XW, Zhao XF, Wang JX. C-type lectin binds to beta-integrin to promote hemocytic phagocytosis in an invertebrate. J Biol Chem (2014) 289:2405-14. doi: 10.1074/jbc.M113.528885

69. Chen DD, Meng XL, Xu JP, Yu JY, Meng MX, Wang J. PcLT, a novel C-type lectin from Procambarus clarkii, is involved in the innate defense against Vibrio alginolyticus and WSSV. Dev Comp Immunol (2013) 39:255-64. doi: 10.1016/j.dci.2012.10.003

70. Liang Z, Yang L, Zheng J, Zuo H, Weng S, He J, et al. A low-density lipoprotein receptor (LDLR) class A domain-containing C-type lectin from Litopenaeus vannamei plays opposite roles in antibacterial and antiviral responses. Dev Comp Immunol (2019) 92:29-34. doi: 10.1016/ j.dci.2018.11.002

Conflict of Interest: The authors declare that the research was conducted in the absence of any commercial or financial relationships that could be construed as a potential conflict of interest.

Copyright $\odot 2021$ Qiu, Chen, Chen, Li, Zhu, Xiong and Wang. This is an open-access article distributed under the terms of the Creative Commons Attribution License (CC BY). The use, distribution or reproduction in other forums is permitted, provided the original author(s) and the copyright owner(s) are credited and that the original publication in this journal is cited, in accordance with accepted academic practice. No use, distribution or reproduction is permitted which does not comply with these terms. 